\title{
Molecular Evolution across Mouse Spermatogenesis
}

\author{
EMILY E. K. KOPANIA ${ }^{1}$, ERICA L. LARSON² ${ }^{2}$ COLIN CALLAHAN ${ }^{1}$, SARA KEEBLE ${ }^{1}$, JEFFREY M. GOOD ${ }^{1}$
}

\begin{abstract}
Genes involved in spermatogenesis tend to evolve rapidly, but we still lack a clear understanding of how different components of molecular evolution vary across this complex developmental process. We used fluorescence activated cell sorting (FACS) to generate expression data for both early (meiotic) and late (postmeiotic) cell types across thirteen inbred strains of mice (Mus) spanning $\sim 7$ million years of evolution. We used these comparative developmental data to investigate the evolution of lineage-specific expression, protein-coding sequences, and expression levels. We found increased lineage specificity and more rapid protein-coding and expression divergence during late spermatogenesis, suggesting that signatures of rapid testis molecular evolution are punctuated across sperm development. Despite strong overall developmental parallels in these components of molecular evolution, protein and expression divergences were only weakly correlated across genes. We detected more rapid protein evolution on the $\mathrm{X}$ chromosome relative to the autosomes, while X-linked gene expression tended to be relatively more conserved likely reflecting chromosome-wide regulatory constraints. Using allele-specific FACS expression data from crosses between four strains, we found that the relative contributions of different regulatory mechanisms also differed between cell-types. Genes showing cis-regulatory changes were more common late in spermatogenesis, and tended to be associated with larger differences in expression levels and greater expression divergence between species. In contrast, genes with trans-acting changes were more common early and tended to be more conserved across species. Our findings advance understanding of gene evolution across spermatogenesis and underscore the fundamental importance of developmental context in molecular evolutionary studies.
\end{abstract}

\section{Introduction}

Mature sperm are the most morphologically diverse animal cell type, likely as a consequence of intense selection on sperm form and function (Pitnick, et al. 2009). Genes involved in spermatogenesis also tend to evolve rapidly (Swanson, et al. 2003; Good and Nachman 2005; Turner, et al. 2008; Larson, et al. 2016; Finseth and Harrison 2018), suggesting that pervasive sexual selection also shapes molecular evolution (Swanson and Vacquier 2002; Harrison, et al. 2015). However, direct genotype-tophenotype connections remain elusive for primary sexually selected traits, and there are additional evolutionary forces acting during spermatogenesis that shape overall patterns of molecular evolution (Good and Nachman 2005; Burgoyne, et al. 2009; Dean, et al. 2009; Larson, et al. 2016; Schumacher and Herlyn 2018). For example, many spermatogenesis genes are highly specialized (Eddy 2002; Chalmel, et al. 2007; Green, et al. 2018), which can relax pleiotropic constraint and contribute to rapid evolution even in the absence of positive directional selection (Winter, et al. 2004; Larracuente, et al. 2008; Meisel 2011). Other components of spermatogenesis are highly conserved because small disruptions can lead to infertility (Burgoyne, et al. 2009). Thus, spermatogenesis genes are likely to experience strong and sometimes contradictory evolutionary pressures. Understanding how these processes interact to shape molecular evolution across spermatogenesis is essential to understanding how natural selection shapes the genetic determinants of male fertility.

There are many components or levels of molecular evolution, spanning from protein sequence changes to differences in gene expression level, timing, and developmental specificity (King and Wilson 1975; Wray, et al. 2003; Larracuente, et al. 2008; Kaessmann 2010; Piasecka, et al. 2013; Cridland, et al. 2020). Many of these components have been shown to evolve relatively rapidly during spermatogenesis (Meiklejohn, et al. 2003; Khaitovich, et al. 2005; Voolstra, et al. 2007; Brawand, et al. 2011; Harrison, et al. 2015; Vicens, et al. 2017; Cridland,

\footnotetext{
${ }^{1}$ DIVISION OF BIOLOGICAL SCIENCES, UNIVERSITY OF MONTANA, MISSOULA, MT 59812, USA

2 DEPARTMENT OF BIOLOGICAL SCIENCES, UNIVERSITY OF DENVER, DENVER, CO 80208, USA

CORRESPONDING AUTHOR(S): JEFFREY M. GOOD JEFFREY.GOOD@UMONTANA.EDU; EMILY E. K. KOPANIA EMILY.KOPANIA@UMCONNECT.UMT.EDU
} 
et al. 2020; Sánchez-Ramírez, et al. 2021), and generally trend towards increased divergence during the later stages of development (Good and Nachman 2005; Piasecka, et al. 2013; Larson, et al. 2016). Novel genes disproportionately arise with testis-specific expression (Levine, et al. 2006; Zhao, et al. 2014; Schroeder, et al. 2019; Cridland, et al. 2020; Lange, et al. 2021), likely as a consequence of the more permissive regulatory environment of the later stages of sperm development (Kaessmann 2010; Soumillon, et al. 2013). Likewise, the later stages of spermatogenesis tend to be enriched for novel testis-specific genes (Eddy 2002; Chalmel, et al. 2007; Green, et al. 2018). These developmental signatures of novelty and specialization are further reflected in patterns of increased divergence of protein sequences (Good and Nachman 2005;

Kousathanas, et al. 2014) and expression levels (Larson, et al. 2016) between species during the later stages of sperm development. Parallel signatures of rapid molecular evolution likely reflect both relaxed constraints during the late stages of spermatogenesis, and enhanced positive selection on late-developing sperm phenotypes (Eddy 2002; Good and Nachman 2005; Larracuente, et al. 2008; Larson, et al. 2016; Cutter and Bundus 2020). However, it remains unclear how strongly different forms of molecular evolution are correlated. For example, changes in gene expression may often be cell or stage-specific and therefore may be less pleiotropic than protein-coding changes. This pleiotropic constraint hypothesis primarily applies to cisregulatory changes, which likely affect one gene, whereas trans-regulatory changes can affect many genes across multiple cell types (Wray, et al. 2003; Carroll 2008; Cutter and Bundus 2020).

The $\mathrm{X}$ chromosome provides a compelling example of how the conflicting selective pressures acting on spermatogenesis may shape different components of molecular evolution. Theory predicts that the $X$ chromosome should evolve more rapidly than the autosomes due to a lower effective population size (i.e., increased genetic drift) and more efficient selection resulting from hemizygosity in males, which immediately exposes recessive beneficial and deleterious mutations to selection (Charlesworth, et al. 1987; Vicoso and Charlesworth 2009). Consistent with these predictions, protein-coding evolution tends to be faster on the $\mathrm{X}$ chromosome compared to the autosomes in several taxa, and this effect is often strongest for genes with male-biased expression (Khaitovich, et al. 2005; Baines and Harr 2007; Baines, et al. 2008; Meisel and Connallon 2013; Parsch and Ellegren 2013; Larson, et al. 2016). Novel genes tend to arise more often on the $X$ chromosome, and these are often expressed during spermatogenesis (Levine, et al. 2006; Kaessmann 2010). There is also some evidence for rapid expression evolution on the $\mathrm{X}$ chromosome in flies and mammals (Khaitovich, et al. 2005; Brawand, et al. 2011; Meisel, et al. 2012; Coolon, et al. 2015), but X-linked expression in mice appears conserved relative to autosomal genes expressed during the later stages of spermatogenesis (Larson, et al. 2016). Stage-specific differences in relative rates of expression evolution on the $X$ chromosome may result from the unique regulatory pattern that the sex chromosomes undergo during mammalian spermatogenesis. In males, the $X$ chromosome is inactivated early in meiosis (i.e., meiotic sex chromosome inactivation, MSCI; McKee and Handel 1993) and remains partially repressed during the postmeiotic haploid stages of sperm development (i.e., postmeiotic sex chromosome repression, PSCR; Namekawa, et al. 2006).

These stage-specific patterns highlight the importance of studying specific components of molecular evolution in a developmental framework (fig. 1A; Larson, et al. 2018a; Cutter and Bundus 2020). However, studies of molecular evolution have primarily focused on pairwise contrasts across nuanced aspects of tissue development (Good and Nachman 2005; Larson, et al. 2016), or examined protein-coding versus regulatory evolution in whole tissues (Khaitovich, et al. 2005; Voolstra, et al. 2007; Larson, et al. 2016; Mack, et al. 2016; Vicens, et al. 2017; Cridland, et al. 2020), without combining both in a phylogenetic framework. Relying on whole tissue expression comparisons may be particularly problematic for spermatogenesis, because differences in testis composition are expected to evolve rapidly between species (Ramm and Schärer 2014; Yapar, et al. 2021) and may confound patterns of expression level divergence (Good, et al. 2010; Larson, et al. 2016; Hunnicutt, et al. 2021). Nonetheless, collection of stage or cell-specific expression data remains technically demanding (da Cruz, et al. 2016; Green, et al. 2018), likely limiting widespread use in comparative studies. As a consequence, most evolutionary studies of gene expression have relied on whole tissue comparisons between closely-related species pairs, instead of using more powerful phylogenetic approaches (Rohlfs and Nielsen 2015; Dunn, et al. 2018).

In this study, we use a comparative developmental approach to gain a more comprehensive understanding of molecular evolution across spermatogenesis in house mice (Mus). Mice are the predominant laboratory model for mammalian reproduction (Phifer-Rixey and Nachman 2015; Firman 2020), with abundant genomic resources (Keane, et al. 2011; Thybert, et al. 2018), and established wild-derived inbred strains that can be crossed to resolve mechanisms underlying expression divergence (i.e., cis- versus transregulatory changes; Mack, et al. 2016). Mice also show divergence in sperm head morphologies across closely related species (Skinner, et al. 2019) and experience sperm competition in the wild (Dean, et al. 2006), providing a compelling system for understanding the evolution of spermatogenesis.

We used fluorescence activated cell sorting (FACS) to resolve patterns of gene expression in two enriched spermatogenic cell populations across several mouse strains, species, and cross-types (fig. 1A). Our study used two main comparisons. First, we evaluated divergence in spermatogenic protein sequences and gene expression levels across thirteen inbred strains of mice, including two subspecies of the house mouse (Mus musculus) and two other Mus species spanning seven million years of evolution (fig. 1B; Chevret, et al. 2005). Second, we used published data from reciprocal crosses between a subset of these inbred strains to resolve the relative contribution of cis- versus trans-regulatory changes to expression 
divergence. We used these data to address five main questions: (i) Is gene expression more lineage-specific during late spermatogenesis? (ii) Do protein-coding sequences and gene expression levels evolve faster during the later stages of spermatogenesis? (iii) Is the rate of molecular evolution elevated on the $X$ chromosome compared to the autosomes, and does this relationship change across spermatogenesis? (iv) To what extent are protein-coding and gene expression divergence correlated, and does this relationship change across developmental stages? (v) Are there differences in the relative contributions of regulatory mechanisms (cis- versus trans-regulatory changes) across spermatogenesis?

\section{Results}

\section{Spermatogenesis Gene Expression by Cell Type and Lineage}

We collected spermatogenesis expression data from 34 mice representing four different species or subspecies: Mus musculus musculus, Mus musculus domesticus, Mus spretus, and Mus pahari. We will use the abbreviations mus, dom, spr, and pah to reference the four major groups, and refer to all taxa as "lineages" for concision (fig. 1B). For each sample, we generated expression data for two spermatogenic cell types, an early meiotic cell type (leptotene-zygotene cells from early prophase of

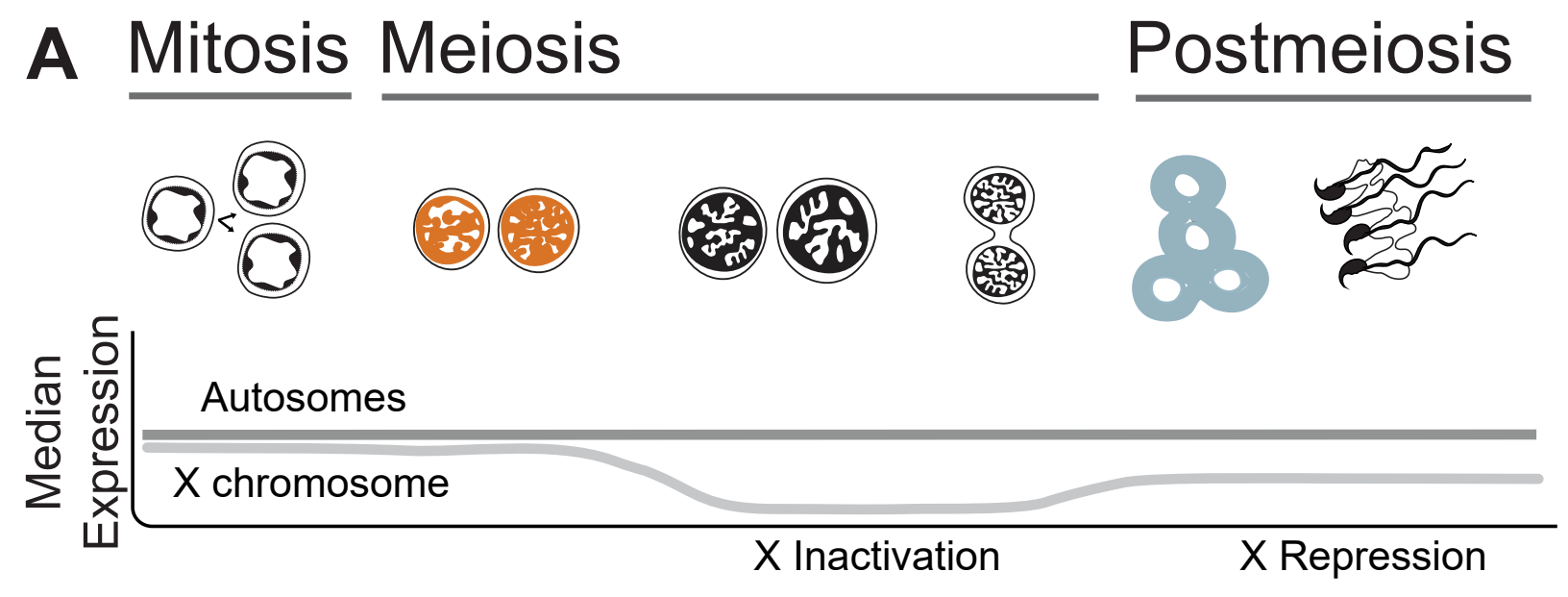

\section{Specificity of genes expressed}
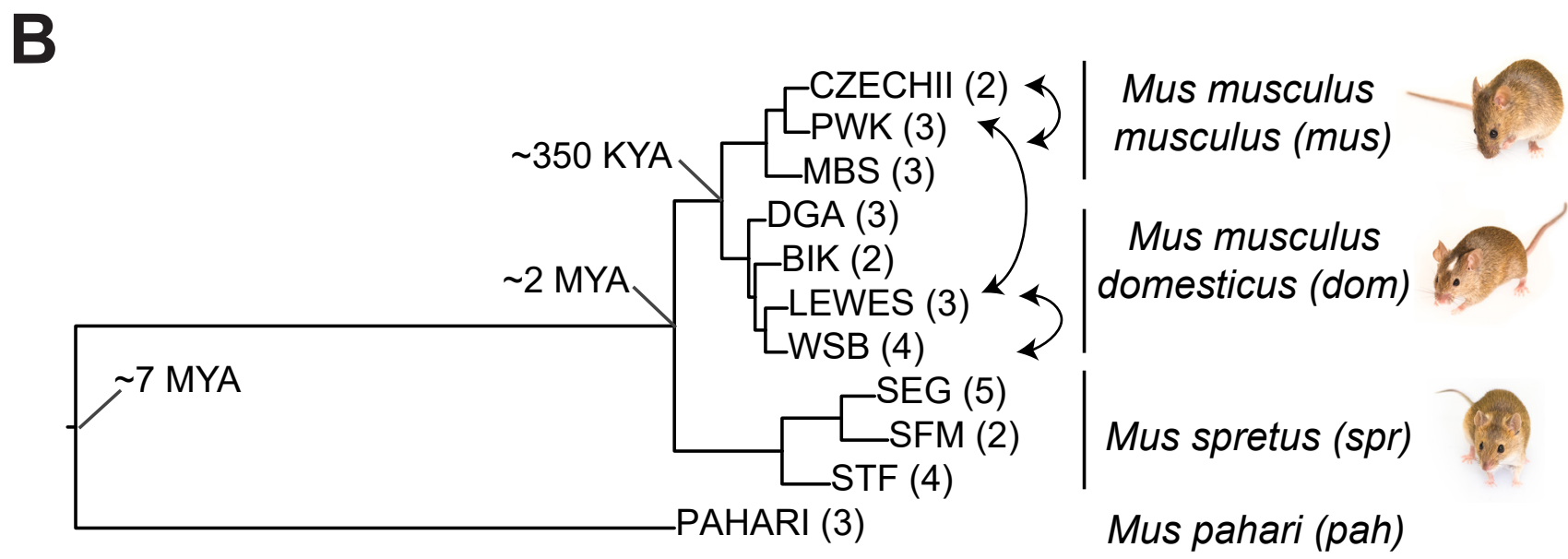

Figure 1. (A) Predictive framework depicting the major stages of spermatogenesis and expected relative expression levels of the $X$ chromosome and autosomes at each stage (Namekawa, et al. 2006). The two cell populations used in this study are leptotene-zygotene ("early", second from left, orange) and round spermatids ("late", second from right, blue). The relative thickness of the gray bar represents the predicted cell type specificity at each stage (Eddy 2002; Chalmel, et al. 2007; Larson, et al. 2016; Green, et al. 2018). (B) Maximum likelihood tree of concatenated exome data from the four Mus species or subspecies used in this study: Mus musculus musculus (mus), Mus musculus domesticus (dom), Mus spretus (spr), Mus pahari (pah). Tips are labeled with the inbred strains from each lineage, with select crosses used to generate F1 hybrids indicated with arrows. Number of individuals sampled for each strain indicated in parentheses. Approximate divergence times are placed at each major node (Chevret, et al. 2005). All nodes had 100\% bootstrap support. 
meiosis I, hereafter "early") and a post-meiotic cell type (round spermatids, hereafter "late"). We identified 23,164 one-to-one orthologs, including both protein-coding and non-protein-coding genes, that were annotated in all four mouse lineages and the mouse reference (GRCm38). From this set, we defined expressed genes as those with an FPKM $>1$ in all samples of a given cell type. Expression variance cleanly separated samples by cell type and lineage (supplementary fig. S1, Supplementary Material online), indicating successful enrichment of different cell types. Most expressed genes were detected in both cell types (table 1). However, approximately one third of the detected genes were preferentially expressed or "induced" in a given cell type (transcripts with $>2 \mathrm{X}$ median expression level in one cell type across all lineages; table 1). We also identified expressed genes that show testis-specific expression based on published multi-tissue expression data (Chalmel, et al. 2007). We found that 493 testis-specific genes were induced late, while only 65 testis-specific genes were induced early (table 1), consistent with increased specificity late in spermatogenesis (Eddy 2002; Larson, et al. 2016;
Green, et al. 2018). To distinguish experimental noise from biologically meaningful expression, we also used a Bayesian approach to determine if a gene was "active" in a tissue or cell type (Thompson, et al. 2020) and found broad overlap with genes in the expressed dataset (table 1). Using the same framework, we identified genes showing evidence for lineage-specific expression ("active" in a single lineage or subset of lineages).

We found that lineage-specificity was rare overall, but more common for autosomal genes active during late spermatogenesis (Pearson's $X 2$ test; dom: $P<<0.0001$, mus: $\mathrm{P}<<0.0001$, spr: $\mathrm{P}<<0.0001$, dom-mus common ancestor: $P<<0.0001$; fig. $2 A$ ). X-linked genes showed no significant differences in lineage-specificity between early and late cell types (fig. 2B), which could reflect a lack of specialization on the sex chromosomes, or reduced power to detect differences between cell types given small sample sizes. Few genes were lineage-specific in both cell types, and all were autosomal (dom: 9 genes, mus: 24 genes, spr: 24 genes, dom-mus: 21 genes). We found similar results using a log fold-change (logFC) approach

Table 1. Counts of genes in each dataset and spermatogenic cell type. Numbers in parentheses represent the percent of genes in the "active" datasets that were also in the "expressed" dataset. TS = testis-specific (inferred from Chalmel, et al. 2007)

\begin{tabular}{c|c|c|c} 
& $\begin{array}{c}\text { early } \\
\text { (spermatocytes) }\end{array}$ & $\begin{array}{c}\text { late } \\
\text { (round spermatids) }\end{array}$ & both early and late \\
\hline expressed & 9570 & 8986 & 7670 \\
\hline induced & 3375 & 2769 & 0 \\
\hline testis-specific (TS) & 544 & 655 & 524 \\
\hline induced and TS & 65 & 493 & 6 \\
\hline active (dom) & $8206(98.2 \%)$ & $8581(90.4 \%)$ & 7289 \\
\hline active (mus) & $8782(97.5 \%)$ & $10098(83.4 \%)$ & 7227 \\
\hline active (spr) & $8728(97.1 \%)$ & $9509(86.0 \%)$ & 6682
\end{tabular}

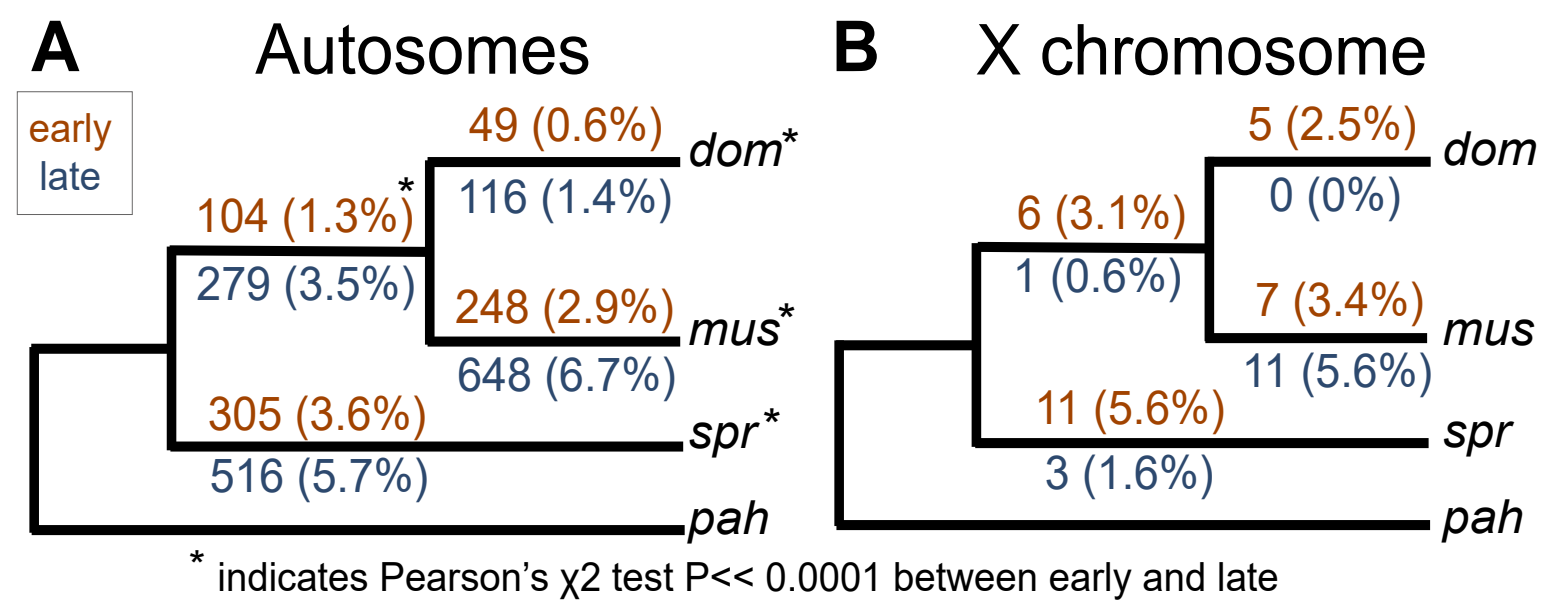

Figure 2. Number of genes that were lineage-specific on each internal branch of the mouse phylogeny used in this study. Numbers in parentheses are the percent of active genes that were lineage-specific. Results are presented separately for the autosomes $(A)$ and $\mathrm{X}$ chromosome $(B)$. Orange values above each branch represent the early cell type and blue values below represent the late cell type. Asterisks indicate a significant difference between early and late on that branch based on a Pearson's $\mathrm{x} 2$ test. 
to identify lineage-specific genes (supplementary fig. S2, Supplementary Material online). These results suggest that lineage-specific expression of spermatogenic genes is relatively uncommon at these shallow phylogenetic scales, but more likely to arise later in spermatogenesis.

\section{Greater Protein-Coding and Gene Expression Divergence during Late Spermatogenesis}

Having detected subtle increases in lineage-specificity late in spermatogenesis, we next tested if rates of protein sequence evolution (dN/dS) and expression level divergence were also elevated during the postmeiotic stage, as has been reported previously (Larson, et al. 2016). Genes induced late in spermatogenesis showed significantly higher rates of protein-coding divergence on both the autosomes $(n=2046$ genes induced early, median $\mathrm{dN} / \mathrm{dS}=0.11 ; \mathrm{n}=1711$ genes induced late, median $\mathrm{dN} /$ $\mathrm{dS}=0.20$; Wilcoxon rank sum test $\mathrm{P}<<0.0001)$ and the $X$ chromosome $(n=54$ genes induced early, median $d N / d S$ $=0.25 ; \mathrm{n}=61$ genes induced late, median $\mathrm{dN} / \mathrm{dS}=0.41$; Wilcoxon rank sum test $P=0.049$; fig. $3 A$, supplementary tables S1 and S2, Supplementary Material online). The 489 testis-specific genes showed elevated dN/dS overall, but most testis-specific genes were expressed in both cell types and there was no significant difference between genes expressed early and late for the autosomes $(n=$ 350 genes expressed early, median $\mathrm{dN} / \mathrm{dS}=0.28 ; n=424$ genes expressed late, median $\mathrm{dN} / \mathrm{dS}=0.30$; Wilcoxon rank sum test $P=1)$ or the $X$ chromosome $(n=16$ genes expressed early; median $\mathrm{dN} / \mathrm{dS}=0.59 ; \mathrm{n}=24$ genes expressed late, median $\mathrm{dN} / \mathrm{dS}=0.58$; Wilcoxon rank sum test $P=1$ ). However, 348 testis-specific genes were preferentially expressed in the late cell type, representing $\sim 20 \%$ of all genes induced late for which we were able to calculate $\mathrm{dN} / \mathrm{dS}$. Taken together, these results confirm that tissue specificity plays an important role in the rapid proteincoding divergence of spermatogenic genes, and that most of this signature involves genes induced during postmeiotic spermatogenesis.

We used a phylogenetic ANOVA to estimate expression divergence while controlling for phylogenetic relatedness and variance within lineages [i.e., the Expression Variance and Evolution (EVE) model; Rohlfs and Nielsen 2015]. We report expression divergence from EVE as $-\log \left(\right.$ beta $\left._{i}\right)$, where beta $i$ is a metric from EVE that represents the ratio of within-lineage variance to betweenlineage evolutionary divergence, and higher positive $-\log \left(\right.$ beta $\left._{i}\right)$ values correspond to greater divergence between lineages.

Expression divergence was higher for genes induced late in spermatogenesis on both the autosomes $(n=2461$ genes induced early, median EVE divergence $=$ $-1.09 ; n=2305$ genes induced late, median EVE divergence $=-0.70$; Wilcoxon rank sum test $P \ll 0.0001)$ and $X$ chromosome $(n=44$ genes induced early, median EVE divergence $=-2.04 ; n=68$ genes induced late, median EVE divergence $=-0.80$; Wilcoxon rank sum test $P=0.00019$; fig. 3B). This pattern held for all expressed genes and testisspecific genes (supplementary table S3, Supplementary Material online). We also found higher divergence late for expressed and induced autosomal genes (supplementary table S3, Supplementary Material online) based on pairwise expression divergences using logFC and the metric from Meisel, et al. (2012); however, the pairwise framework did not give a consistent pattern on the $\mathrm{X}$ chromosome. When looking at all genes, most pairwise comparisons showed higher divergence late, but induced genes showed no difference between early and late spermatogenesis for most comparisons. However, the dom versus spr comparison had
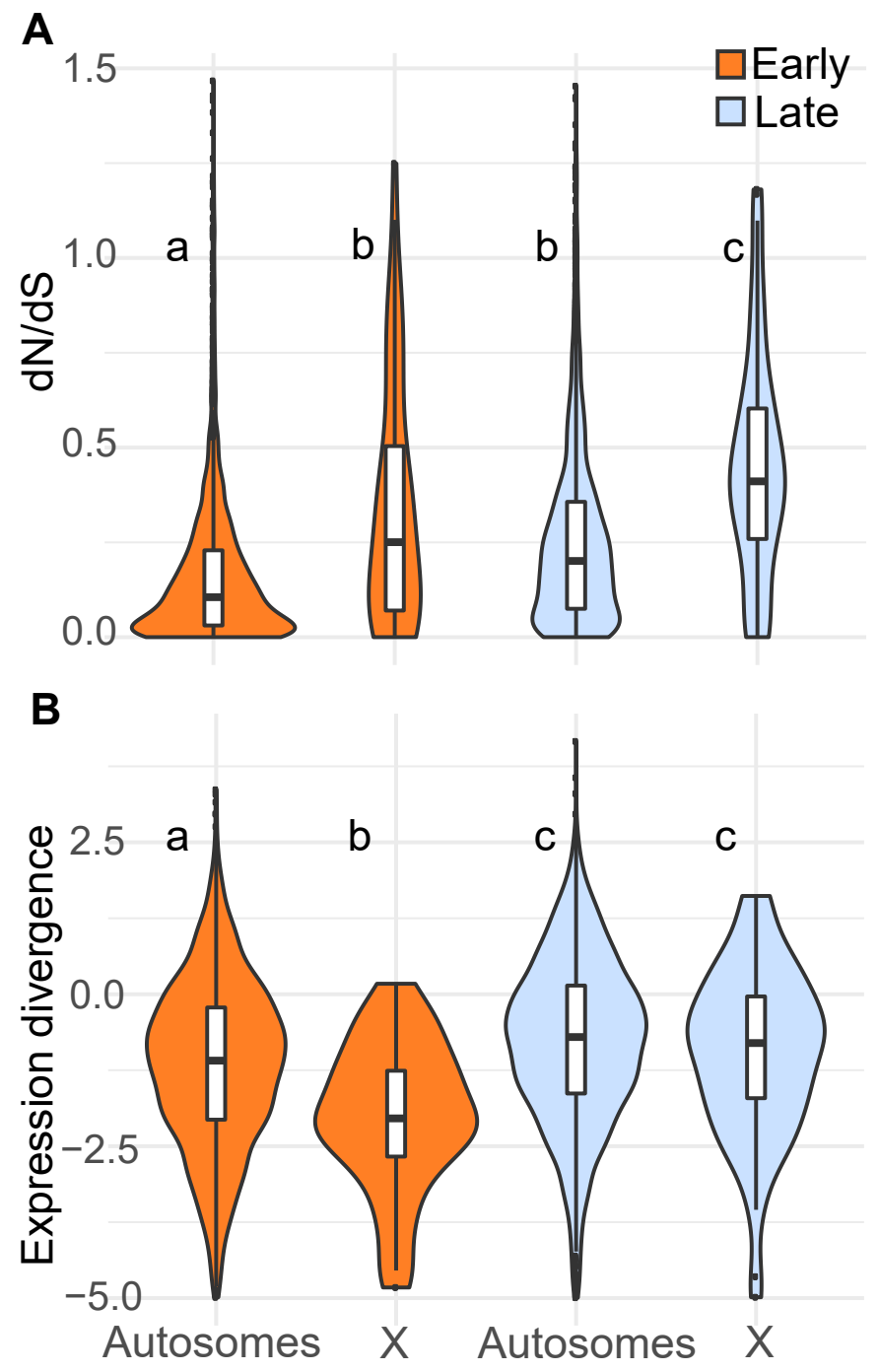

Figure 3. $(A)$ Protein-coding and $(B)$ expression divergence on the autosomes and $\mathrm{X}$ chromosome for genes induced in each cell type. Expression divergence values on the $y$-axis are $-\log \left(\right.$ beta $\left._{i}\right)$, where beta $_{i}$ is the measure of expression divergence from EVE. Higher values on the $y$-axis represent higher divergence. The center of each violin plot is a standard boxplot, with the center horizontal line representing the median divergence value. The violins show the probability density of divergence values for each group. A wider part of the violin at a given value means genes expressed in that group are more likely to have that divergence value. The letters above each violin indicate significant differences between the cell types and chromosome types based on a Wilcoxon rank-sum test. 
lower divergence late for all expressed genes and induced genes (supplementary table S3, Supplementary Material online). Collectively, we found strong evidence for more rapid protein-coding and gene expression level divergence during postmeiotic spermatogenesis, suggesting that these general patterns hold after controlling for phylogeny and at deeper divergence levels than had previously been shown in mice (Larson, et al. 2016). Despite our expanded phylogenetic sample, we still lacked the power to determine if more rapid expression and protein-coding divergence is due to positive directional selection (supplementary fig. S3, Supplementary Material online).

\section{Weak Positive Correlation between Gene Expression and Protein-Coding Divergence}

We next tested for more general relationships between protein-coding and expression divergence across sets of genes expressed or induced during spermatogenesis (supplementary fig. S4, supplementary table S4, Supplementary Material online). Across all autosomal genes expressed early, there was a weak positive correlation between $\mathrm{dN} / \mathrm{dS}$ and pairwise expression divergence ( $\rho$ $=0.13-0.17$, Spearman's rank correlation $\mathrm{P}<0.0001$ ). For induced genes, this correlation was weaker but still significant $(\rho=0.07-0.11$, Spearman's rank correlation $P<0.05)$. For the late cell type, there was also a weak positive correlation between pairwise expression divergence and $\mathrm{dN} / \mathrm{dS}$ on the autosomes, but the correlation was weaker than that seen in the early cell type $(\rho=0.03-$ 0.05 , Spearman's rank correlation $P<0.05$ ). There was no correlation for the set of genes induced late. When looking only at genes with evidence for positive directional selection at the protein-coding level after correction for multiple tests (366 genes), the correlation was stronger on the autosomes late for the dom vs $\operatorname{spr}(\mathrm{n}=250$ genes, $\rho=0.17$, Spearman's rank correlation $P=0.02$ ) and mus vs spr comparisons ( $n=249$ genes, $\rho=0.18$, Spearman's rank correlation $P<0.0001$ ). When comparing $\mathrm{dN} / \mathrm{dS}$ to EVE expression divergence, we only saw a significant positive correlation for genes expressed late that were also under positive selection at the protein-coding level $(n=160$ genes, $\rho=0.18$, Spearman's rank correlation $P=0.04$ ). In summary, we tended to observe a positive relationship between protein-coding and expression level divergence, but the strength of this relationship was weak and varied by gene set and divergence metric.

\section{Faster-X Protein-Coding but Not Gene Expression Evolution}

In addition to comparisons between spermatogenesis cell types, we compared relative rates of molecular evolution between $X$-linked and autosomal genes within a cell type. We found that protein-coding divergence was higher on the $\mathrm{X}$ chromosome, both early and late, across all gene sets (fig. 3A, supplementary tables S1 and S2, Supplementary Material online) consistent with several previous studies (Khaitovich, et al. 2005; Baines, et al. 2008; Meisel and Connallon 2013; Kousathanas, et al. 2014; Larson, et al. 2016). For expression evolution, we found lower divergence on the $X$ chromosome early using EVE $(n=$ 2461 autosomal genes, median EVE divergence $=-1.09$; $\mathrm{n}=44 \mathrm{X}$-linked genes, median EVE divergence $=-2.04$; Wilcoxon rank sum test $P=0.00015$; fig. 3B), but higher $X$-linked divergence when using pairwise comparisons (supplementary table S3, Supplementary Material online). A major difference between these approaches was that EVE calculates divergence across a phylogeny, so genes that show divergent expression levels in one lineage may still be conserved across the entire phylogeny. We detected significant correlations between pairwise divergence values for different pairwise comparisons on the autosomes, and during late spermatogenesis, but lower or non-significant correlations on the $\mathrm{X}$ early (table 2 ). Thus, many genes on the $\mathrm{X}$ chromosome expressed early showed relatively high divergence between two particular lineages, but lower divergence across other pairwise comparisons and across the phylogeny as a whole. This lineage-specific variance underscores the importance of evaluating gene expression divergence in a phylogenetic framework (Rohlfs and Nielsen 2015; Dunn, et al. 2018).

In late spermatogenic cells (i.e., round spermatids), $X$-linked expression divergence was similar to or lower than on the autosomes depending on the contrast and approach. Using EVE, we found similar divergence on the $X$ chromosome and autosomes late $(n=2305$ autosomal genes, median EVE divergence $=-0.70 ; n=68 X$-linked genes, median EVE divergence $=-0.80$; Wilcoxon rank sum test $P=0.34$; fig. $3 B$ ), while pairwise comparisons gave mixed results, depending on which two lineages were compared (supplementary table S3, Supplementary Material online). There were proportionally fewer differentially expressed genes on the X chromosome (fig. 4, supplementary fig. S5, Supplementary Material online), and this pattern was strongest for the more closely related comparisons (hypergeometric test; mus versus dom $\mathrm{P}<<$ 0.0001 , spr versus dom $\mathrm{P}<<0.0001$, spr versus mus $\mathrm{P}$ $<0.0001)$. Across all metrics of expression divergence and both developmental stages, there was no evidence for pervasive faster- $X$ gene expression level evolution.

\section{Relative Contributions of cis- and trans-Regulatory Evolution Vary across Spermatogenesis}

Having shown differences in expression divergence between cell types, we next asked if there were differences in the types of regulatory mutations (e.g., cis- versus transregulatory changes) underlying expression divergence in each cell type. We used whole testis (Mack, et al. 2016) and FACS-sorted (Larson, et al. 2017) data from reciprocal crosses between house mouse subspecies (dom $\mathrm{x}$ mus) to estimate allele-specific expression (ASE) and assign genes to eight different regulatory categories: cis, trans, cis X trans, compensatory, cis + trans opposite, cis + trans same, other, and conserved (Coolon, et al. 2014; Mack, et al. 2016).

Across all cell types and genotypes, $50-90 \%$ of genes were conserved. Comparing the two spermatogenic stages, we saw striking differences in the proportions of non-conserved genes within each regulatory category (fig. 5 , supplementary table S5, Supplementary Material online). 
Table 2. Correlation between pairwise expression divergence values for all possible pairwise comparisons.

Numbers presented are $\rho$ values from a Spearman's rank correlation test. We tested for correlations in pairwise expression divergence value among induced genes in each stage and chromosome group (early X, early autosomal, late X, and late autosomal). Gray boxes indicate no significant correlation between pairwise divergence values after FDR correction (Spearman's rank correlation $\mathrm{P}>0.05$ ). Bolded values indicate the lowest Spearman's $\rho$ value for each pairwise comparison across the four stages and chromosome groups.

\begin{tabular}{|c|c|c|c|c|c|c|}
\hline & & dom vs mus & dom vs spr & mus vs spr & dom vs pah & mus vs pah \\
\hline Early, & dom vs spr & 0.34 & & & & \\
\hline \multirow[t]{4}{*}{ X-linked } & mus vs spr & 0.07 & 0.28 & & & \\
\hline & dom vs pah & 0.07 & 0.14 & 0.19 & & \\
\hline & mus vs pah & 0.16 & 0.10 & 0.03 & 0.62 & \\
\hline & spr vs pah & 0.14 & 0.27 & 0.16 & 0.58 & 0.67 \\
\hline Early, & dom vs spr & 0.32 & & & & \\
\hline \multirow[t]{4}{*}{ autosomal } & mus vs spr & 0.32 & 0.61 & & & \\
\hline & dom vs pah & 0.28 & 0.28 & 0.27 & & \\
\hline & mus vs pah & 0.29 & 0.26 & 0.30 & 0.74 & \\
\hline & spr vs pah & 0.24 & 0.32 & 0.34 & 0.55 & 0.57 \\
\hline Late, & dom vs spr & 0.36 & & & & \\
\hline \multirow[t]{4}{*}{ X-linked } & mus vs spr & 0.50 & 0.45 & & & \\
\hline & dom vs pah & 0.20 & 0.23 & 0.22 & & \\
\hline & mus vs pah & 0.28 & 0.28 & 0.36 & 0.74 & \\
\hline & sprvs pah & 0.15 & 0.20 & 0.20 & 0.73 & 0.72 \\
\hline Late, & dom vs spr & 0.35 & & & & \\
\hline \multirow[t]{4}{*}{ autosomal } & mus vs spr & 0.37 & 0.59 & & & \\
\hline & dom vs pah & 0.30 & 0.33 & 0.30 & & \\
\hline & mus vs pah & 0.30 & 0.30 & 0.33 & 0.76 & \\
\hline & spr vs pah & 0.25 & 0.32 & 0.33 & 0.64 & 0.63 \\
\hline
\end{tabular}
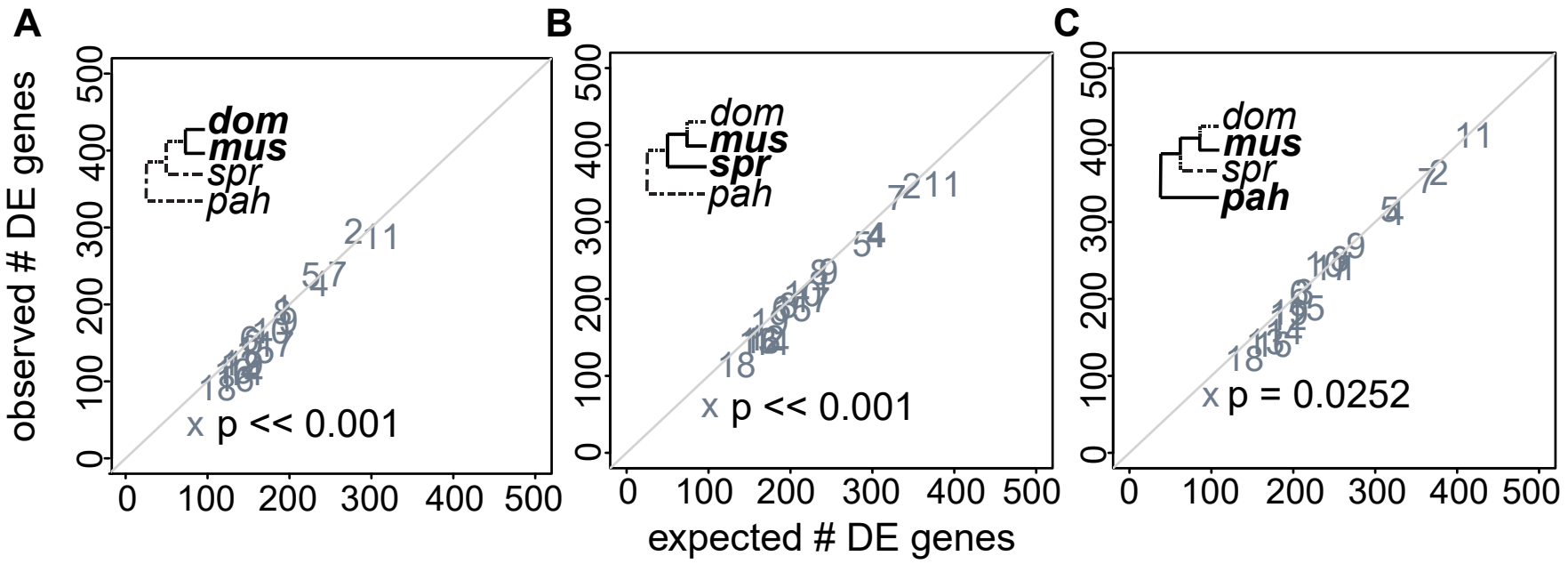

Figure 4. Observed versus expected number of genes differentially expressed (DE) in late spermatogenesis for three pairwise comparisons at different levels of evolutionary divergence: $(A)$ dom versus mus, $(B)$ spr versus mus, and $(C)$ pah versus mus. Each point represents a different chromosome. The diagonal line is the one-to-one line at which the observed number of DE genes equals the expected number. P-values are shown for the $X$ chromosome only. They are based on a hypergeometric test for enrichment and corrected for multiple tests using a false discovery rate correction. A significant $p$-value indicates that the observed number of DE genes is different from the expected number. 
Trans was more common than cis early, whereas trans and cis made up a similar proportion of regulatory changes late (fig. 5, supplementary table S5, Supplementary Material online). Compensatory changes (compensatory and cisttrans opposite) were more common than reinforcing (cis+trans same) in both cell types, but there was a higher relative proportion of reinforcing late (fig. 5, supplementary table S5, Supplementary Material online). We focused on results for the dom (LEWES) $q$ X mus (PWK) ${ }^{\lambda}$ cross (fig. 5) because these $F 1$ hybrids are more fertile and therefore less likely to have misexpressed genes due to hybrid incompatibilities (Good, et al. 2010). However, the subfertile reciprocal hybrid genotype also showed similar overall proportions of genes in each regulatory category. The proportions of different regulatory mechanisms in whole testes were more similar to the late cell type (supplementary table S5, Supplementary Material online), consistent with previous studies showing high overlap in expression profiles between whole testes and spermatid stage cells (Soumillon, et al. 2013). We further verified our results using pure strain (LEWES and PWK) expression data from our phylogenetic expression dataset to determine differences in parental strain expression levels (supplementary table S5, Supplementary Material online). Finally, we evaluated the relative contributions of regulatory mechanisms contributing to expression differences between strains within each M. musculus subspecies using expression data from within-subspecies F1s (WSB X LEWES and CZECHII $X P W K$ ) and from the respective parental inbred strains. Consistent with the more divergent F1 hybrids, there was more trans than cis early but some variation depending on subspecies and cross-type (supplementary table S5, Supplementary Material online). In summary, early and late spermatogenesis differed in the types of regulatory mutations contributing to expression divergence, with a proportionally higher contribution of trans-regulatory changes early. This pattern was consistent across different degrees of evolutionary divergence and between reciprocal crosses.

\section{cis-Regulatory Changes Tended to Have Larger Effects on Expression Level Divergence}

Given that trans-regulatory changes were proportionally more common during early spermatogenesis (fig. 5), and that expression levels tended to be more conserved early (fig. 3), we hypothesized that trans-regulatory changes would have smaller effect sizes (Coolon, et al. 2014; Hill, et al. 2020). Consistent with this, genes with trans changes showed lower median divergence than those with cis changes (fig. 6). We saw higher divergence for reinforcing mutations based on logFC, but not EVE (fig. 6), suggesting that genes with reinforcing changes specific to the dom and mus comparison may not accumulate more divergence at deeper phylogenetic levels. For the early cell type, $26 \%$ of genes in the reinforcing category overlapped with genes that had high pairwise divergence between dom and mus, whereas only $10-16 \%$ of genes in this category overlapped with high divergence genes in other pairwise comparisons (supplementary table S6, Supplementary Material online). Similar patterns were observed for late cell type genes (supplementary table S6, Supplementary Material online). Collectively, cis-regulatory changes tended to have larger effects on expression divergence than trans-regulatory changes, and reinforcing mutations tended to have large effects on expression divergence between mus and dom, but not at deeper levels of evolutionary divergence.
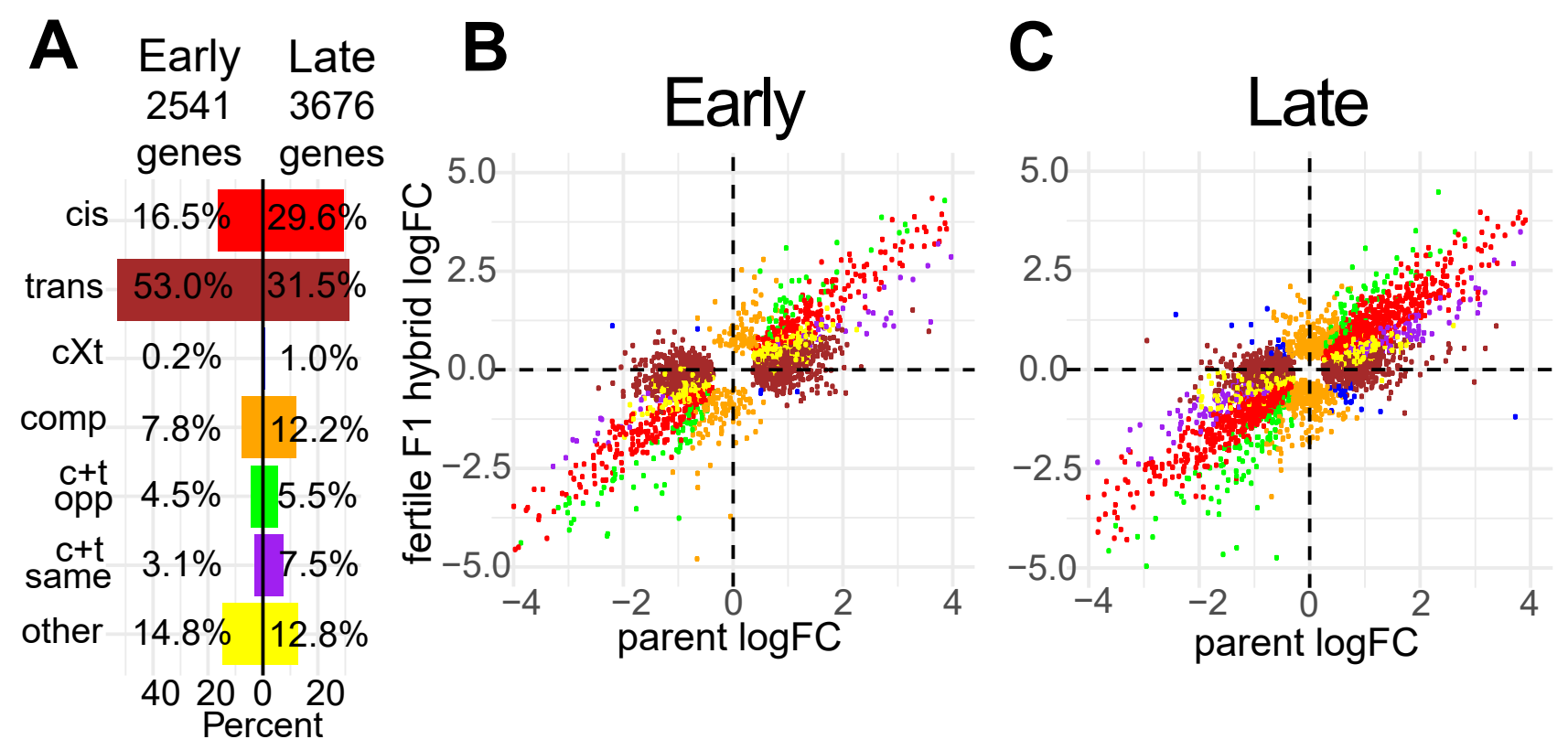

Figure 5. Regulatory category results for the fertile F1 hybrid (LEWES $q$ X PWK $\left.{ }^{\lambda}\right)(A)$ Percent of non-conserved genes in each regulatory category both early and late. $(B$ and $C$ ) Expression logFC between alleles within the fertile $F 1$ (y-axis) plotted against the expression logFC between the parental subspecies ( $\mathrm{x}$-axis). Each point represents a single gene. Colors correspond to $(A)$ and indicate the regulatory category to which that gene was assigned. $\mathrm{cXt}=$ cis $\mathrm{X}$ trans; comp $=$ compensatory; $\mathrm{c}+\mathrm{t}$ opp $=$ cis + trans opposite; $\mathrm{c}+\mathrm{t}$ same $=$ cis + trans same 


\section{Discussion}

Developmental stage and context play an important role in shaping the molecular evolution of reproductive genes (Dean, et al. 2009; Larson, et al. 2016; Finseth and Harrison 2018; Schumacher and Herlyn 2018), with genes expressed in later developmental stages evolving more rapidly (Good and Nachman 2005; Larson, et al. 2016). However, comparing gene expression and protein divergence across developmental stages has rarely been done in a phylogenetic framework. In this study, we combined comparative genomics with cell sorting in four species to understand mouse spermatogenesis evolution across a common developmental framework. Our results give insight into how evolution proceeds at different stages of sperm development, at different molecular levels, and on different chromosome types.

\section{Molecular Divergence across Development}

There is a long-standing prediction that early developmental stages should be more constrained, with evolutionary divergence gradually increasing across development (Abzhanov 2013), which likely contributes to more rapid molecular evolution during the later stages of sperm development. In addition, the postmeiotic stages are enriched for genes with narrower expression profiles or highly specific biological functions and are therefore expected to experience relaxed pleiotropic constraint (Eddy 2002; Good and Nachman 2005; Green, et al. 2018), also motivating our general hypothesis that the postmeiotic round spermatid stage would diverge more rapidly. Sexual selection is also likely to be a primary determinant of spermatogenic evolution, but variation in the intensity of sexual selection across spermatogenesis is not well
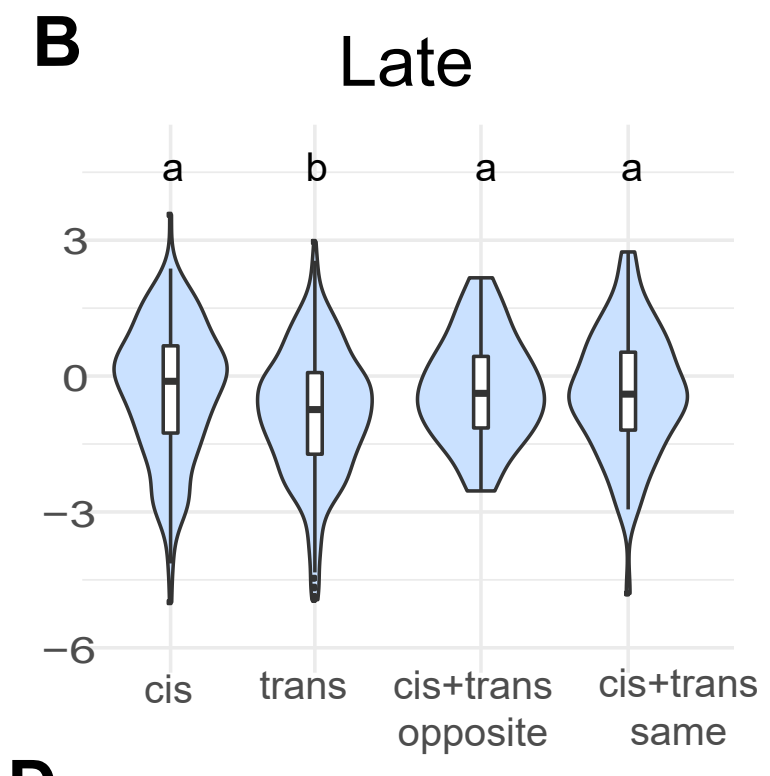

D

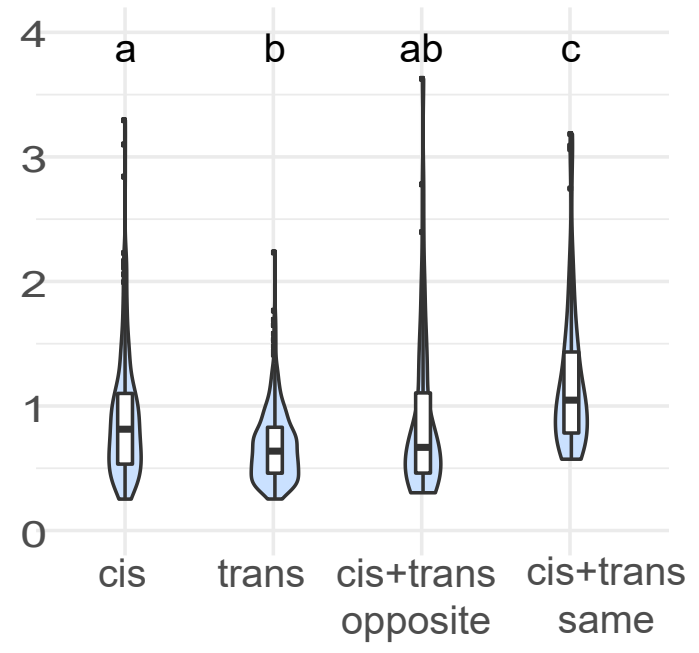

Regulatory Category

Figure 6. Expression divergence violin plots by regulatory category for the fertile hybrid. Expression divergence is calculated using the value from EVE $(A$ and $B)$ and as the absolute value of the logFC in expression between parental subspecies $(C$ and $D$ ). Plots $A$ and $C$ correspond to the early cell type and plots $B$ and $D$ correspond to the late cell type. Letters indicate significant differences between categories based on a pairwise Wilcoxon rank sum test. 
understood (White-Cooper, et al. 2009). Sperm competition and cryptic female choice can select for changes in sperm production rate, form, or function, and many aspects of sperm morphology correlate with the intensity of postmating sexual selection (Lüpold, et al. 2016; McLennan, et al. 2017; Pahl, et al. 2018). Rates of mitotic and initial meiotic divisions during early spermatogenesis can control the overall rate of sperm production (Ramm and Schärer 2014). Therefore, selection for increased sperm production likely acts during the development of spermatogonia, the diploid mitotic cells (White-Cooper, et al. 2009). In contrast, sexual selection shaping the form and function of mature sperm (e.g., sperm swimming speed and fertilization ability) likely acts on later developmental stages such as haploid spermatids (Alavioon, et al. 2017). However, many genes involved in mature spermatozoa functions are also highly expressed during early meiosis (da Cruz, et al. 2016), suggesting that spermatozoa may be shaped by regulatory networks operating throughout spermatogenesis.

All components of molecular evolution that we considered showed more divergence when considering genes induced in late spermatogenesis: lineage-specific expression (fig. 2), protein-coding divergence, and expression level divergence (fig. 3). On first principles, these likely result from a combination of positive selection and relaxed developmental and pleiotropic constraint (Eddy 2002; Swanson and Vacquier 2002; Winter, et al. 2004; Good and Nachman 2005; Abzhanov 2013; Green, et al. 2018). However, our study was underpowered to formally test for positive selection using likelihood ratio test approaches (Anisimova, et al. 2001; Rohlfs and Nielsen 2015). Thus, the relative contributions of positive selection and relaxed constraint to rapid spermatogenesis evolution remain unclear, especially for gene expression phenotypes. Induced genes provided strong evidence for rapid evolution late, but results were less clear when looking at other genes. Spermatogenesis is a transcriptionally complex process, with most of the genome expressed in the testes (Soumillon, et al. 2013) and high overlap between genes expressed early and late in our dataset (table 1). For protein-coding divergence, we saw more rapid evolution late only when looking at the induced dataset, but not when looking at all expressed genes, likely because most genes in our dataset were expressed in both cell types. For expression divergence, there was more rapid evolution late even when looking at all expressed genes. This suggests that even genes with broader (i.e., non-induced) expression patterns tended to show more conserved expression early in spermatogenesis.

Testis-specific genes tended to be both induced late and rapidly evolving at the protein-coding level. Testisspecific and male-biased gene sequences often evolve rapidly, which could be the result of positive selection on genes with specific spermatogenesis functions as well as relaxed constraint because these genes tend to have highly specific functions (Meiklejohn, et al. 2003; Baines, et al. 2008; Meisel 2011; Parsch and Ellegren 2013). However, we did not see a significant faster late pattern for proteincoding or pairwise expression divergence when looking only at testis-specific genes. Although there were relatively few testis-specific genes, it appears that they tended to be rapidly evolving regardless of which spermatogenesis stage they were expressed in. If generally true, more rapid divergence late in spermatogenesis may partially reflect a higher proportion of testis-specific genes induced in the late cell type (table 1).

In addition to these broad patterns of molecular evolution, we explored the potential functional relevance of rapid divergence for specific genes (supplementary table S7, Supplementary Material online). We detected 20 genes with high (>2.5) EVE divergence in either cell type, and of these 15 were broadly expressed, but five may have specific roles in spermatogenesis (The UniProt Consortium 2020). For example, Rnf19a had an EVE value of 4.2 in the late cell type and has a known role in the formation of the sex body, which isolates the sex chromosomes in the nucleus during meiosis, a process that is required for proper spermatogenesis (Párraga and del Mazo 2000) and appears to be disrupted in sterile hybrid mice (Bhattacharyya, et al. 2013).

\section{Gene Expression versus Protein-Coding Divergence} Protein-coding changes alter a gene in every tissue and developmental stage in which it is expressed, whereas expression changes have the potential to be more specific (Wray, et al. 2003; Carroll 2008). Expression changes, specifically cis-regulatory changes, should be less constrained by pleiotropy and may underlie evolutionary changes when purifying selection acts more strongly against protein-coding divergence (Wray, et al. 2003; Carroll 2008). Under this model, we might expect to see less pronounced differences in relative expression levels when comparing early versus late stages. However, more recent work has shown that cis-regulatory elements such as enhancers can be highly pleiotropic, so cis-regulatory changes may be more constrained than once thought (Sabarís, et al. 2019; Hill, et al. 2020). If gene expression and protein-coding are subject to similar constraints, we would expect them to show similar evolutionary patterns across spermatogenesis, as we observed for autosomal genes (fig. 3).

Interestingly, despite parallel trends in relative divergence across spermatogenesis, expression level divergence and protein-coding divergence were not strongly correlated across genes, suggesting that these two types of molecular changes mostly evolve independently (Khaitovich, et al. 2005). Perhaps surprisingly, there was no overlap between genes with very rapid protein-coding divergence (dN/dS > 1.5) and high expression divergence (EVE divergence > 2.5). Likewise, only 26 genes with high pairwise expression divergence in at least one comparison (pairwise divergence metric $>1$ ) also had high proteincoding divergence ( $\mathrm{dN} / \mathrm{dS}>1.5$; supplementary table S7, Supplementary Material online). Whether expression or protein-coding is more rapid for a particular gene may depend on factors such as expression breadth and protein function, but rarely did spermatogenic genes appear to be rapidly evolving for both gene expression and protein sequences.

We also investigated lineage-specificity. Testes and sperm tend to be enriched for lineage-specific genes 
(Brawand, et al. 2011) and novel genes (Schroeder, et al. 2019; Cridland, et al. 2020; Lange, et al. 2021). Lineage-specific and novel genes may be common in spermatogenesis because testes are highly transcriptionally active and have a high tissue-specific expression profile, which may allow new genes to arise without disrupting other processes (Levine, et al. 2006; Kaessmann 2010; Soumillon, et al. 2013; Zhao, et al. 2014). We found that late spermatogenesis also had proportionally more lineagespecific genes (fig. 2). Increased lineage-specificity late is consistent with and likely contributed to higher protein and expression level divergence late, as all results suggest that spermatogenesis can tolerate more genetic changes during the late stages without impacting fertility.

\section{$X$ Chromosome Evolution}

The $\mathrm{X}$ chromosome is predicted to evolve faster than the autosomes because it has a lower effective population size and is therefore more susceptible to drift, and because it is hemizygous in males so beneficial recessive mutations will fix more quickly (Charlesworth, et al. 1987; Vicoso and Charlesworth 2009). Empirical studies show evidence for a faster-X effect at the protein-coding level in many taxa, particularly for male reproductive genes (Khaitovich, et al. 2005; Baines, et al. 2008; Meisel and Connallon 2013; Parsch and Ellegren 2013; Larson, et al. 2016; Whittle, et al. 2020). Our data provide strong evidence for faster-X protein-coding evolution for both early and late spermatogenesis, demonstrating that the faster-X effect applies across genes involved in different spermatogenesis stages in mice.

Our results were more complex for expression evolution, with phylogenetic (Rohlfs and Nielsen 2015) and pairwise approaches (Meisel, et al. 2012) sometimes yielding contrasting results. In the early cell type, pairwise comparisons supported a faster-X effect, while the phylogenetic model did not (fig. 3B, supplementary table S3, Supplementary Material online). Correlations between different pairwise divergence values were relatively low on the $\mathrm{X}$ chromosome early, suggesting that $\mathrm{X}$-linked genes with high expression level divergence in one pairwise comparison did not tend to have high divergence in other comparisons (table 2). In the late cell type, both phylogenetic and pairwise divergence metrics supported a similar rate of $X$-linked and autosomal expression evolution (fig. 3B, supplementary table S3, Supplementary Material online). It is well-established that lineage-specific changes can create false signatures of rapid divergence in pairwise comparisons (Felsenstein 1985), including in studies of gene expression evolution (Dunn, et al. 2018). Thus, our results highlight the importance of accounting for shared evolutionary history when inferring general evolutionary trends (Rohlfs and Nielsen 2015; Dunn, et al. 2018).

Overall, our results did not support a faster-X effect for testis gene expression evolution, in contrast to several previous studies (Khaitovich, et al. 2005; Brawand, et al. 2011; Meisel, et al. 2012). These studies were in other systems and used whole testes samples, which are made up of different cell types, so signals of expression divergence may partially reflect differences in cell type composition rather than true per cell changes in expression levels (Good, et al. 2010; Hunnicutt, et al. 2021; Yapar, et al. 2021). One previous study used cell-type specific data and found that the $X$ chromosome showed fewer differentially expressed genes during late spermatogenesis between mus and dom (Larson, et al. 2016), and our phylogenetic sampling demonstrates that this result likely applies across mouse species.

\section{Contrasting patterns of expression level and} protein sequence evolution on the $\mathrm{X}$ chromosome during spermatogenesis suggest that these two levels of molecular evolution experience different evolutionary pressures (Larson, et al. 2016). Theoretical predictions for the faster-X effect on protein-coding evolution may also apply to gene expression changes, but only for cis-regulatory changes where the causative mutations are on the $X$ chromosome (Meisel and Connallon 2013; Larson, et al. 2016). During spermatogenesis, the sex chromosomes undergo $\mathrm{MSCl}$ and PSCR, which likely imposes an overall repressive regulatory environment that constrains gene expression levels but not protein-coding changes. Disruption of $\mathrm{MSCl}$ and PSCR strongly impairs male fertility, so evolutionary constraints on $\mathrm{X}$ chromosome expression during spermatogenesis are expected to be strong (Burgoyne, et al. 2009; Good, et al. 2010; Larson, et al. 2017). Our results support the hypothesis that unique $\mathrm{X}$-linked regulatory constraints reduce expression level divergence during spermatogenesis, while still allowing rapid protein-coding divergence (Larson, et al. 2016; Larson, et al. 2018a). This finding underscores how different components of molecular evolution may experience unique evolutionary pressures that result in distinct patterns of divergence (Brawand, et al. 2011; Halligan, et al. 2013; Larson, et al. 2016).

\section{Regulatory Mechanisms Underlying Expression Divergence}

Resolving the relative contributions of cis- versus transacting mutations underlying expression divergence is an important step towards understanding the genetic architecture of expression phenotypes and how different evolutionary forces may act on gene expression (Benowitz, et al. 2020; Hill, et al. 2020). Although considerable progress has been made in a few key model systems on this important question (Goncalves, et al. 2012; Coolon, et al. 2014; Mack, et al. 2016; Benowitz, et al. 2020; Cridland, et al. 2020; Sánchez-Ramírez, et al. 2021), available data mostly come from whole tissues or organisms. Our results showed that the relative contribution of underlying regulatory mechanisms can differ dramatically between two cell types within a single complex tissue. While these striking differences are perhaps an expected consequence of different selective pressures acting on cellular function and developmental stage, they also underscore how difficult it is to resolve regulatory phenotypes from complex tissues.

As cis-regulatory changes are generally thought to be less pleiotropic (Carroll 2008; Hill, et al. 2020), we predicted that cis-regulatory mutations would be proportionally more common in early spermatogenesis, but we found the opposite pattern (fig. 5, supplementary table S5, Supplementary Material online). The relative 
contributions of cis- and trans-regulatory changes to expression divergence likely depend on other factors, including a tendency of cis mutations to have larger individual effect sizes (Coolon, et al. 2014; Hill, et al. 2020). We did observe proportionally more cis-regulatory changes of large effect during late spermatogenesis (fig. 6D) underlying higher overall expression divergence at this stage (fig. 3). Thus, differences in individual effect sizes of cis- versus trans-acting changes likely play a central role in shaping regulatory evolution across mouse spermatogenesis.

Cis- and trans-regulatory mutations can combine to affect the expression of a single gene, either in the same direction (reinforcing) or in opposite directions (compensatory; Goncalves, et al. 2012; Coolon, et al. 2014; Mack, et al. 2016). We observed a higher proportion of compensatory mutations than reinforcing mutations across both spermatogenesis cell types and in whole testes. This was expected given that gene expression tends to evolve under stabilizing selection (Rohlfs and Nielsen 2015), and it is consistent with previous studies across many tissue types in mice (Goncalves, et al. 2012; Mack, et al. 2016), flies (Coolon, et al. 2014; Benowitz, et al. 2020), and roundworms (Sánchez-Ramírez, et al. 2021). We also saw relatively more reinforcing mutations during postmeiotic spermatogenesis. Reinforcing mutations tended to have a larger effect size based on expression logFC between mus and dom (fig. 6D), thus large-effect reinforcing changes also likely contribute to higher expression level divergence in late spermatogenesis.

Given the striking differences that we saw between just two cell types, it is likely that complex tissues composed of many cell types may often give different results than isolated cell populations. Consistent with this prediction, our observed proportions of genes in each regulatory category differ from some other published results in house mouse whole tissues (i.e., liver, Goncalves, et al. 2012; whole testes, Mack, et al. 2016), primarily in that we saw a much higher proportion of genes in the trans category. We also found some different patterns when reanalyzing whole testes expression data from Mack, et al. (2016) that likely reflect technical differences in the analytical pipelines used between studies (supplementary table S5, see Supplementary Methods for details, Supplementary Material online). In general, our analysis used more conservative approaches to test for significant DE or ASE. Thus, only genes showing relatively pronounced differences in expression levels between genotypes or alleles were assigned to regulatory mechanisms in our study.

We also found that the relative proportion of cis- and trans-regulatory changes were similar between whole testes and the late cell type in the fertile F1 hybrid (supplementary table S5, Supplementary Material online), consistent with the observation that postmeiotic spermatids have a disproportionately large contribution to mouse whole testes expression patterns (Hunnicutt, et al. 2021). These results suggest that changes in the relative intensities of different selective pressures acting across spermatogenesis not only change the extent of expression level divergence, but also select for different mechanisms of regulatory evolution underlying these expression changes. Given this, analyzing such patterns at the level of whole organisms or tissues seems unlikely to provide a clear understanding of how mechanisms of regulatory evolution proceed in underlying cells. Indeed, even enriched cell populations as we have generated may be limited by relative purities.

By considering both expression divergence across the Mus phylogeny and underlying mechanisms of regulatory divergence between two lineages (mus and $d o m$ ), our study also provided a novel opportunity to connect different types of regulatory changes to patterns of expression divergence at a deeper phylogenetic scale. Although trans-acting changes were relatively common (fig. 5), genes with cis-regulatory changes between mus and dom tended to have higher phylogeny-wide expression divergence than those with trans-regulatory changes for both cell types (fig. 6A, 6B). This suggests that genes showing cis-regulatory changes are also more likely to accumulate regulatory differences over time, resulting in phylogeny-wide expression divergence, whereas genes showing trans-regulatory changes at relatively shallow evolutionary scales tend to be relatively conserved across the Mus phylogeny. Genes with reinforcing changes also had relatively low phylogeny-wide expression level divergence (fig. 6A, 6B), in contrast to their high pairwise divergence between mus and dom (fig. 6C, 6D). Genes in this category likely have large-effect, lineage-specific changes in expression that may be under purifying selection over deeper phylogenetic levels. Finally, our phylogenetic contrast revealed rapid expression level divergence late in spermatogenesis. By combining these data with allele-specific expression data, we further showed that cis-regulatory changes are likely to underlie this rapid phylogeny-wide expression divergence in late spermatogenesis.

\section{Materials and Methods}

\section{Mouse Resources}

We investigated gene expression and protein-coding evolution in 12 Mus musculus domesticus (dom) individuals from four inbred strains (2 BIK/g, 3 DGA, 3 LEWES/EiJ, 4 WSB/EiJ), 8 M. m. musculus (mus) individuals from three inbred strains (2 CZII/EiJ, 3 MBS, 3 PWK/PhJ), $11 \mathrm{M}$. spretus (spr) individuals from three inbred strains (5 SEG, 2 SFM, 4 STF), and 3 M. pahari (pah) individuals from one inbred strain (3 PAHARI/EiJ; fig. 1B). By using multiple wildderived inbred strains of dom, mus, and spr, we sampled natural within-species variation while also having biological replicates of genetically similar individuals. These mice were maintained in breeding colonies at the University of Montana (UM) Department of Laboratory Animal Resources (IACUC protocol 002-13). These colonies were initially established from mice purchased from The Jackson Laboratory, Bar Harbor, ME (CZECHII/EiJ, PWK/PhJ, WSB/ EiJ, LEWES/EiJ, PAHARI/EiJ) or acquired from Matthew Dean's colonies at University of Southern California which were derived from François Bonhomme's stocks at the University of Montpellier, Montpellier, France (MBS, BIK, 
DGA, STF, SFM, SEG). We weaned males at $\sim 21$ days postpartum (dpp) into same sex sibling groups and caged males individually at least 15 days prior to euthanization to avoid dominance effects on testes expression. We euthanized mice at $60-160$ dpp by $\mathrm{CO} 2$ followed by cervical dislocation.

For expression data from reciprocal F1 males, we used FACS enriched expression data from (Larson, et al. 2017). These data include males from reciprocal F1 crosses between different inbred strains within each M. musculus subspecies (mus: CZECHII females X PWK males, dom: WSB females X LEWES males), as well as reciprocal mus and dom $\mathrm{F} 1$ hybrids (LEWES females $\mathrm{X}$ PWK males and PWK females X LEWES males), allowing us to compare results at two different levels of divergence (i.e., within and between lineages). We also analyzed whole testes expression data from Mack, et al. (2016) to compare FACS enriched cell-types to whole testes, including crosses between different strains within each $M$. musculus subspecies (LEWES females X WSB males and PWK females X CZII males) and the same reciprocal F1 hybrid crosses to those in (Larson, et al. 2017).

\section{Testis Cell Sorting and RNAseq}

We collected testes from mice immediately following euthanization and isolated cells at different stages of spermatogenesis using Fluorescence Activated Cell Sorting (FACS; Getun, et al. 2011). The full FACS protocol is available on GitHub (https://github.com/goodest-goodlab/ good-protocols/tree/main/protocols/FACS). Briefly, we decapsulated testes and washed them twice with $1 \mathrm{mg} /$ $\mathrm{mL}$ collagenase (Worthington Biochemical), $0.004 \mathrm{mg} /$ $\mathrm{mL}$ DNase I (Qiagen), and GBSS (Sigma), followed by disassociation with $1 \mathrm{mg} / \mathrm{mL}$ trypsin (Worthington Biochemical) and $0.004 \mathrm{mg} / \mathrm{mL}$ DNase I. We then inactivated trypsin with $0.16 \mathrm{mg} / \mathrm{mL}$ fetal calf serum (Sigma). For each wash and disassociation step, we incubated and agitated samples at $33^{\circ} \mathrm{C}$ for 15 minutes on a VWR minishaker at $120 \mathrm{rpm}$. We stained cells with $0.36 \mathrm{mg} / \mathrm{mL}$ Hoechst 33324 (Invitrogen) and $0.002 \mathrm{mg} / \mathrm{mL}$ propidium iodide, filtered with a $40 \mu \mathrm{m}$ cell filter, and sorted using a FACSAria llu cell sorter (BD Biosciences) at the UM Center for Environmental Health Sciences Fluorescence Cytometry Core. We periodically added $0.004 \mathrm{mg} / \mathrm{mL}$ DNase I as needed during sorting to prevent DNA clumps from clogging the sorter. We sorted cells into $15 \mu \mathrm{L}$ beta-mercaptoethanol (Sigma) per $1 \mathrm{~mL}$ of RLT lysis buffer (Qiagen) and kept samples on ice whenever they were not in the incubator or the cell sorter. For this study, we focused on two cell populations: early meiotic spermatocytes (leptotene/ zygotene) and postmeiotic round spermatids. We extracted RNA using the Qiagen RNeasy Blood and Tissue Kit and checked RNA integrity with a Bioanalyzer 2000 (Agilent) or TapeStation 2200 (Agilent). All samples except one had RIN $\geq 7$ (supplementary table S8, Supplementary Material online). We prepared RNAseq libraries using the Agilent SureSelect protocol and sequenced samples at the Hudson Alpha Institute for Biotechnology using Illumina NextSeq (75bp single end).

\section{Mus strain phylogeny}

We generated the phylogeny in fig. 1B using available exome (Chang, et al. 2017; Sarver, et al. 2017) and whole genome (Keane, et al. 2011; Thybert, et al. 2018) sequence data (PRJNA326865, PRJNA323493, PRJEB2003,

PRJEB14896). Genotypes were based on iterative mapping assemblies relative to the house mouse reference genome (mm10) conducted using pseudo-it v3.0 (Sarver, et al. 2017; https://github.com/goodest-goodlab/pseudo-it/tree/kopaniaetal-2021) that restricts genotyping to targeted exons. We ran pseudo-it with one iteration to generate consensus fasta files for each sample. We then extracted exons, aligned these regions using MAFFT v7.271 (Katoh and Standley 2013), converted to PHYLIP format using AMAS (Borowiec 2016), and inferred a maximum likelihood concatenated tree using IQ-TREE v2.1.4-beta (Nguyen, et al. 2014).

\section{Processing and Quantification of Gene Expression Data}

We used $R$ version 3.6.3 and Bioconductor version 3.10 for all analyses. We trimmed raw reads for adaptors and low-quality bases using expHTS (Streett, et al. 2015) and mapped trimmed reads with TopHat version 2.1.0 (Kim, et al. 2013). Genome assemblies were previously published for all four lineages (Keane, et al. 2011; Thybert, et al. 2018), allowing us to map reads to the correct assembly and reduce reference bias (Sarver, et al. 2017). Mapping rates were consistent across lineages (supplementary table S8, Supplementary Material online). To select orthologous genes among the four lineages, we used BiomaRt (Durinck, et al. 2005; Durinck, et al. 2009) to identify one-to-one Ensembl orthologs and retained only those that were present in all genome assemblies and the mouse reference build GRCm38.

We used edgeR 3.28.1 (Robinson, et al. 2010) to normalize expression data, calculate fragments per kilobase per million reads (FPKM), and perform differential expression (DE) analyses. A gene was defined as "expressed" in our dataset if it had an FPKM > 1 in at least eight samples. A gene was expressed in a particular lineage and cell type if it had an FPKM > 1 in all samples of that lineage and cell type. A gene was considered induced in a particular cell type if its median FPKM in that cell type across all lineages was greater than two times its median FPKM in the other cell type across all lineages. Testisspecific genes were those only expressed in testis based on the mouse tissue expression data from (Chalmel, et al. 2007).

We defined lineage-specific genes in two ways. First, we used a log fold change (logFC) method in which a gene was considered lineage-specific if its median expression level in a lineage was greater than two times its median expression level in any of the other three lineages. Second, we used a Bayesian approach to determine if a gene is active or inactive in an expression dataset based on transcript levels as implemented with the program Zigzag (Thompson, et al. 2020). Genes identified as being active (posterior $P>0.5$ ) in one lineage and inactive (posterior $P<0.5)$ in the other lineages were considered lineagespecific. We ran Zigzag twice and only included genes with consistent active or inactive assignments between 
the two runs. Both the logFC and Zigzag analyses were performed for each cell type, so a gene could be lineagespecific in one cell type but not the other. For each lineage, we determined the proportion of expressed (logFC) or active (Zigzag) genes that were lineage-specific and used a Pearson's $X 2$ test to determine if one cell type had greater lineage-specificity than the other.

\section{Protein-Coding Divergence}

We used the "iqtree-omp" command in IQTree version 1.5.5 (Nguyen, et al. 2014) to infer a mouse species tree based on gene trees estimated from the reference sequences for all four mouse lineages (Keane, et al. 2011; Thybert, et al. 2018). We took the longest transcript for all one-to-one orthologs and aligned these using MAFFT v7.271 (Katoh and Standley 2013) and converted to PHYLIP format using AMAS (Borowiec 2016). We used a custom script to exclude genes that did not begin with a start codon, had early stop codons, or had sequence lengths that were not multiples of three. We then used the Codeml program in the PAML package to calculate protein-coding divergence and test for positive selection on protein-coding genes (Yang 2007). We used the M0 model to calculate phylogeny-wide dN/dS for each gene, which we report as the overall proteincoding divergence values. We also performed a likelihood ratio test between the M8 and M8a site-based models to test for positive directional selection on each gene (Swanson, et al. 2003).

\section{Differential Expression}

We performed all analyses of expression level divergence for three different gene sets: expressed genes, induced genes, and testis-specific genes. To calculate expression divergence in a phylogenetic framework, we used the EVE model (Rohlfs and Nielsen 2015), which performs a phylogenetic ANOVA using an Ornstein-Uhlenbeck model to evaluate divergence while controlling for evolutionary relatedness. We report expression divergence from EVE as $-\log \left(\right.$ beta $\left._{i}\right)$, where beta $i$ is a metric from EVE that represents the ratio of within-lineage variance to between-lineage evolutionary divergence. By taking the negative log, higher positive numbers correspond to greater evolutionary divergence. We excluded genes with extremely low divergence values $\left[-\log \left(\right.\right.$ beta $\left.\left._{i}\right)<-5\right]$ because this subset did not show a linear relationship between evolutionary divergence and population variance and therefore violated underlying assumptions of the EVE model (supplementary fig. S6, Supplementary Material online).

We also calculated expression divergence in a pairwise framework (Meisel, et al. 2012). This method takes the difference in expression level between two lineages and normalizes based on the average expression of the gene in both lineages:

$$
D_{a, i j}=\frac{\left|S_{a, i}-S_{a, j}\right|}{\left(S_{a, i}+S_{a, j}\right) / 2}
$$

$\mathrm{Da}, \mathrm{ij}$ is the divergence of gene a between lineages $\mathrm{i}$ and $j$. Sa, is the median FPKM of gene a in lineage $i$, and $\mathrm{Sa}, \mathrm{j}$ is the median FPKM of gene a in lineage $\mathrm{j}$. We also calculated the logFC in expression between every pairwise comparison of lineages as an additional pairwise divergence metric (Robinson, et al. 2010). For the EVE, pairwise divergence, and logFC methods, we compared relative expression divergence between cell types and between the $\mathrm{X}$ chromosome and autosomes using a Wilcoxon rank-sum test. We tested if certain cell types or chromosome types showed greater correlation among pairwise divergence values using Spearman's rank correlation.

We also compared relative expression divergence on the $\mathrm{X}$ chromosome versus the autosomes using the proportion of DE genes on each chromosome (Good, et al. 2010; Larson, et al. 2016). First, we calculated the proportion of expressed genes that are DE across all autosomes. We then multiplied this proportion by the number of genes expressed on each chromosome to calculate the expected number of DE genes for each chromosome. We plotted the observed number of DE genes against the expected number and used a hypergeometric test to evaluate if each chromosome is over- or underenriched for DE genes.

\section{Allele-Specific Expression and Regulatory Divergence} We used the modtools and lapels-suspenders pipelines (Huang, et al. 2014) to reduce mapping bias and to assign the parental origin of reads in F1 individuals (See Supplementary Methods for details, Supplementary Material online). This approach requires mapping to pseudogenomes generated using modtools to resolve differences in genome coordinates between different references. We used published pseudogenomes for WSB and PWK, which incorporate single nucleotide variants (SNVs) and indels from these strains into the GRCm38 mouse reference build (Huang, et al. 2014). For LEWES and CZECHII, we generated our own pseudogenomes with modtools version 1.0.2 using published VCF files (Morgan, et al. 2016; Larson, et al. 2018b). We developed a custom pipeline (See Supplementary Methods for details, Supplementary Material online) to assign genes to regulatory categories following previous recommendations (Coolon, et al. 2014; Mack, et al. 2016; Combs and Fraser 2018; Benowitz, et al. 2020). To determine significant differences between cell types, we performed a Pearson's $x 2$ test followed by false discovery rate correction for multiple tests.

\section{Competing Interests}

The authors declare they have no conflict of interest.

\section{Data Availability}

RNAseq data generated for this project are available through the National Center for Biotechnology Information under accession PRJNA735780. Individual sample accessions are in supplementary table S8. A table of genes in our analyses and whether they were considered expressed, induced, or active in each cell type is available in supplementary table S9. 


\section{Author contributions}

J.M.G conceived and funded the project. E.E.K.K., E.L.L., and J.M.G. designed the experiments. E.L.L. and S.K. performed the mouse husbandry and breeding experiments. C.C., S.K., and E.L.L. performed mouse dissections and cell sorts. C.C. and E.L.L. prepared the sequencing libraries. E.E.K.K. analyzed the data. E.E.K.K., E.L.L., and J.M.G. wrote the manuscript with input from all authors.

\section{Acknowledgements}

We would like to thank Pamela K. Shaw and the UM Fluorescence Cytometry Core supported by an Institutional Development Award from the NIGMS (P30GM103338), the UM Genomics Core supported by the M.J. Murdock Charitable Trust, the UM Lab Animal Resources staff, Gregg Thomas for assistance generating the phylogeny in fig. 1B, Nathanael Herrera for mouse photos, and the Good Lab for helpful advice. This work was supported by grants from the Eunice Kennedy Shriver National Institute of Child Health and Human Development of the National Institutes of Health (R01-HD073439, R01-HD094787 to JMG). EEKK was supported by the National Science Foundation Graduate Research Fellowship Program (DGE-1313190). ELL was supported by the National Science Foundation (DEB-2012041). Any opinions, findings, and conclusions or recommendations expressed in this material are those of the author(s) and do not necessarily reflect the views of the National Science Foundation or the National Institutes of Health.

\section{References}

Abzhanov A. 2013. von Baer's law for the ages: lost and found principles of developmental evolution. Trends Genet. 29:712-722.

Alavioon G, Hotzy C, Nakhro K, Rudolf S, Scofield DG, Zajitschek S, Maklakov AA, Immler S. 2017. Haploid selection within a single ejaculate increases offspring fitness. Proc Natl Acad Sci U S A. 114:8053-8058.

Anisimova M, Bielawski JP, Yang Z. 2001. Accuracy and Power of the Likelihood Ratio Test in Detecting Adaptive Molecular Evolution. Mol Biol Evol. 18:1585-1592.

Baines JF, Harr B. 2007. Reduced X-Linked Diversity in Derived Populations of House Mice. Genetics. 175:1911-1921.

Baines JF, Sawyer SA, Hartl DL, Parsch J. 2008. Effects of X-Linkage and Sex-Biased Gene Expression on the Rate of Adaptive Protein Evolution in Drosophila. Mol Biol Evol. 25:1639-1650.

Benowitz KM, Coleman JM, Allan CW, Matzkin LM. 2020. Contributions of cis- and trans-Regulatory Evolution to Transcriptomic Divergence across Populations in the Drosophila mojavensis Larval Brain. Genome Biol Evol. 12:1407-1418.

Bhattacharyya T, Gregorova S, Mihola O, Anger M, Sebestova J, Denny P, Simecek P, Forejt J. 2013. Mechanistic basis of infertility of mouse intersubspecific hybrids. Proc Natl Acad Sci U S A. 110:E468-E477.

Borowiec ML. 2016. AMAS: a fast tool for alignment manipulation and computing of summary statistics. PeerJ. 4:e1660.

Brawand D, Soumillon M, Necsulea A, Julien P, Csardi G, Harrigan P, Weier M, Liechti A, Aximu-Petri A, Kircher M, et al. 2011. The evolution of gene expression levels in mammalian organs. Nature. 478:343-348.

Burgoyne PS, Mahadevaiah SK, Turner JMA. 2009. The consequences of asynapsis for mammalian meiosis. Nat Rev Genet. 10:207-216.

Carroll SB. 2008. Evo-Devo and an Expanding Evolutionary Synthesis: A Genetic Theory of Morphological Evolution. Cell. 134:25-36.

Chalmel F, Rolland AD, Niederhauser-Wiederkehr C, Chung SSW, Demougin P, Gattiker A, Moore J, Patard J-J, Wolgemuth DJ, Jégou
B, et al. 2007. The conserved transcriptome in human and rodent male gametogenesis. Proc Natl Acad Sci U S A. 104:8346-8351.

Chang PL, Kopania E, Keeble S, Sarver BAJ, Larson E, Orth A, Belkhir K, Boursot P, Bonhomme F, Good JM, et al. 2017. Whole exome sequencing of wild-derived inbred strains of mice improves power to link phenotype and genotype. Mamm Genome. 28:416-425.

Charlesworth B, Coyne JA, Barton NH. 1987. The Relative Rates of Evolution of Sex Chromosomes and Autosomes. Am Nat. 130:113146.

Chevret P, Veyrunes F, Britton-Davidian J. 2005. Molecular phylogeny of the genus Mus (Rodentia: Murinae) based on mitochondrial and nuclear data. Biol J Linn Soc. 84:417-427.

Combs PA, Fraser HB. 2018. Spatially varying cis-regulatory divergence in Drosophila embryos elucidates cis-regulatory logic. PLoS Genet. 14:e1007631.

Coolon JD, McManus CJ, Stevenson KR, Graveley BR, Wittkopp PJ. 2014. Tempo and mode of regulatory evolution in Drosophila. Genome Res. 24:797-808.

Coolon JD, Stevenson KR, McManus CJ, Yang B, Graveley BR, Wittkopp PJ. 2015. Molecular Mechanisms and Evolutionary Processes Contributing to Accelerated Divergence of Gene Expression on the Drosophila X Chromosome. Mol Biol Evol. 32:2605-2615.

Cridland JM, Majane AC, Sheehy HK, Begun DJ. 2020. Polymorphism and Divergence of Novel Gene Expression Patterns in Drosophila melanogaster. Genetics. 216:79-93.

Cutter AD, Bundus JD. 2020. Speciation and the developmental alarm clock. eLife. 9:e56276.

da Cruz I, Rodríguez-Casuriaga R, Santiñaque FF, Farías J, Curti G, Capoano CA, Folle GA, Benavente R, Sotelo-Silveira JR, Geisinger A. 2016. Transcriptome analysis of highly purified mouse spermatogenic cell populations: gene expression signatures switch from meiotic-to postmeiotic-related processes at pachytene stage. BMC Genomics. 17:294.

Dean MD, Ardlie KG, Nachman MW. 2006. The frequency of multiple paternity suggests that sperm competition is common in house mice (Mus domesticus). Mol Ecol. 15:4141-4151.

Dean MD, Clark NL, Findlay GD, Karn RC, Yi X, Swanson WJ, MacCoss MJ, Nachman MW. 2009. Proteomics and Comparative Genomic Investigations Reveal Heterogeneity in Evolutionary Rate of Male Reproductive Proteins in Mice (Mus domesticus). Mol Biol Evol. 26:1733-1743.

Dunn CW, Zapata F, Munro C, Siebert S, Hejnol A. 2018. Pairwise comparisons across species are problematic when analyzing functional genomic data. Proc Natl Acad Sci U S A. 115:E409-E417.

Durinck S, Moreau Y, Kasprzyk A, Davis S, De Moor B, Brazma A, Huber W. 2005. BioMart and Bioconductor: a powerful link between biological databases and microarray data analysis. Bioinformatics. 21:3439-3440.

Durinck S, Spellman PT, Birney E, Huber W. 2009. Mapping identifiers for the integration of genomic datasets with the R/Bioconductor package biomaRt. Nat Protoc. 4:1184-1191.

Eddy EM. 2002. Male germ cell gene expression. Recent Prog Horm Res. 57:103-128.

Felsenstein J. 1985. Phylogenies and the Comparative Method. Am Nat. 125:1-15.

Finseth FR, Harrison RG. 2018. Genes Integral to the Reproductive Function of Male Reproductive Tissues Drive Heterogeneity in Evolutionary Rates in Japanese Quail. G3-Genes Genom Genet. 8:39-51.

Firman RC. 2020. Of mice and women: advances in mammalian sperm competition with a focus on the female perspective. Philos Trans R Soc Lond, Ser B: Biol Sci. 375:20200082.

Getun IV, Torres B, Bois PRJ. 2011. Flow Cytometry Purification of Mouse Meiotic Cells. JoVE-J Vis Exp.2602.

Goncalves A, Leigh-Brown S, Thybert D, Stefflova K, Turro E, Flicek P, Brazma A, Odom DT, Marioni JC. 2012. Extensive compensatory cis-trans regulation in the evolution of mouse gene expression. Genome Res. 22:2376-2384.

Good JM, Giger T, Dean MD, Nachman MW. 2010. Widespread Over-Expression of the $X$ Chromosome in Sterile F1 Hybrid Mice. PLoS Genet. 6:e1001148.

Good JM, Nachman MW. 2005. Rates of Protein Evolution Are Positively Correlated with Developmental Timing of Expression During Mouse Spermatogenesis. Mol Biol Evol. 22:1044-1052.

Green CD, Ma Q, Manske GL, Shami AN, Zheng X, Marini S, Moritz L, Sul- 
bioRxiv preprint doi: https://doi.org/10.1101/2021.08.04.455131; this version posted August 25, 2021. The copyright holder for this preprint (which was not certified by peer review) is the author/funder. All rights reserved. No reuse allowed without permission.

tan C, Gurczynski SJ, Moore BB, et al. 2018. A Comprehensive Roadmap of Murine Spermatogenesis Defined by Single-Cell RNA-Seq. Dev Cell.

Halligan DL, Kousathanas A, Ness RW, Harr B, Eöry L, Keane TM, Adams DJ, Keightley PD. 2013. Contributions of Protein-Coding and Regulatory Change to Adaptive Molecular Evolution in Murid Rodents. PLoS Genet. 9:e1003995.

Harrison PW, Wright AE, Zimmer F, Dean R, Montgomery SH, Pointer MA, Mank JE. 2015. Sexual selection drives evolution and rapid turnover of male gene expression. Proc Natl Acad Sci U S A. 112:4393-4398.

Hill MS, Vande Zande P, Wittkopp PJ. 2020. Molecular and evolutionary processes generating variation in gene expression. Nat Rev Genet.

Huang S, Holt J, Kao C-Y, McMillan L, Wang W. 2014. A novel multi-alignment pipeline for high-throughput sequencing data. Database. 2014.

Hunnicutt KE, Good JM, Larson EL. 2021. unpublished data. https://wwwbiorxivorg/content/101101/20210708451646v1. last accessed July 12, 2021.

Kaessmann H. 2010. Origins, evolution, and phenotypic impact of new genes. Genome Res. 20:1313-1326.

Katoh K, Standley DM. 2013. MAFFT Multiple Sequence Alignment Software Version 7: Improvements in Performance and Usability. Mol Biol Evol. 30:772-780.

Keane TM, Goodstadt L, Danecek P, White MA, Wong K, Yalcin B, Heger A, Agam A, Slater G, Goodson M, et al. 2011. Mouse genomic variation and its effect on phenotypes and gene regulation. Nature. 477:289-294.

Khaitovich P, Hellmann I, Enard W, Nowick K, Leinweber M, Franz H, Weiss G, Lachmann M, Pääbo S. 2005. Parallel Patterns of Evolution in the Genomes and Transcriptomes of Humans and Chimpanzees. Science. 309:1850-1854.

Kim D, Pertea G, Trapnell C, Pimentel H, Kelley R, Salzberg SL. 2013. TopHat2: accurate alignment of transcriptomes in the presence of insertions, deletions and gene fusions. Genome Biol. 14:R36.

King M, Wilson A. 1975. Evolution at two levels in humans and chimpanzees. Science. 188:107-116.

Kousathanas A, Halligan DL, Keightley PD. 2014. Faster-X Adaptive Protein Evolution in House Mice. Genetics. 196:1131-1143.

Lange A, Patel PH, Heames B, Damry AM, Saenger T, Jackson CJ, Findlay GD, Bornberg-Bauer E. 2021. Structural and functional characterization of a putative de novo gene in Drosophila. Nat Commun. 12:1667.

Larracuente AM, Sackton TB, Greenberg AJ, Wong A, Singh ND, Sturgill D, Zhang Y, Oliver B, Clark AG. 2008. Evolution of protein-coding genes in Drosophila. Trends Genet. 24:114-123.

Larson EL, Keeble S, Vanderpool D, Dean MD, Good JM. 2017. The Composite Regulatory Basis of the Large X-Effect in Mouse Speciation. Mol Biol Evol. 34:282-295.

Larson EL, Kopania EEK, Good JM. 2018a. Spermatogenesis and the Evolution of Mammalian Sex Chromosomes. Trends Genet. 34:722-732.

Larson EL, Vanderpool D, Keeble S, Zhou M, Sarver BAJ, Smith AD, Dean MD, Good JM. 2016. Contrasting Levels of Molecular Evolution on the Mouse X Chromosome. Genetics. 203:1841-1857.

Larson EL, Vanderpool D, Sarver BAJ, Callahan C, Keeble S, Provencio LP, Kessler MD, Stewart V, Nordquist E, Dean MD, et al. 2018b. The Evolution of Polymorphic Hybrid Incompatibilities in House Mice. Genetics.

Levine MT, Jones CD, Kern AD, Lindfors HA, Begun DJ. 2006. Novel genes derived from noncoding DNA in Drosophila melanogaster are frequently $\mathrm{X}$-linked and exhibit testis-biased expression. Proc Natl Acad Sci U S A. 103:9935-9939.

Lüpold S, Manier MK, Puniamoorthy N, Schoff C, Starmer WT, Luepold SHB, Belote JM, Pitnick S. 2016. How sexual selection can drive the evolution of costly sperm ornamentation. Nature. 533:535538.

Mack KL, Campbell P, Nachman MW. 2016. Gene regulation and speciation in house mice. Genome Res.

McKee BD, Handel MA. 1993. Sex chromosomes, recombination, and chromatin conformation. Chromosoma. 102:71-80.

McLennan HJ, Lüpold S, Smissen P, Rowe KC, Breed WG. 2017. Greater sperm complexity in the Australasian old endemic rodents (Tribe: Hydromyini) is associated with increased levels of inter-male sperm competition. Reprod Fertil Dev. 29:921-930.

Meiklejohn CD, Parsch J, Ranz JM, Hartl DL. 2003. Rapid evolution of male-biased gene expression in Drosophila. Proc Natl Acad Sci U S A. 100:9894-9899.

Meisel RP. 2011. Towards a More Nuanced Understanding of the Relationship between Sex-Biased Gene Expression and Rates of Protein-Coding Sequence Evolution. Mol Biol Evol. 28:1893-1900.

Meisel RP, Connallon T. 2013. The faster-X effect: integrating theory and data. Trends Genet. 29:537-544.

Meisel RP, Malone JH, Clark AG. 2012. Faster-X Evolution of Gene Expression in Drosophila. PLoS Genet. 8:e1003013.

Morgan AP, Didion JP, Doran AG, Holt JM, McMillan L, Keane TM, Pardo-Manuel de Villena F. 2016. Genome Report: Whole Genome Sequence of Two Wild-Derived Mus musculus domesticus Inbred Strains, LEWES/EiJ and ZALENDE/EiJ, with Different Diploid Numbers. G3-Genes Genom Genet.

Namekawa SH, Park PJ, Zhang L-F, Shima JE, McCarrey JR, Griswold MD, Lee JT. 2006. Postmeiotic Sex Chromatin in the Male Germline of Mice. Curr Biol. 16:660-667.

Nguyen L-T, Schmidt HA, von Haeseler A, Minh BQ. 2014. IQ-TREE: A Fast and Effective Stochastic Algorithm for Estimating Maximum-Likelihood Phylogenies. Mol Biol Evol. 32:268-274.

Pahl T, McLennan HJ, Wang Y, Achmadi AS, Rowe KC, Aplin K, Breed WG. 2018. Sperm morphology of the Rattini - are the interspecific differences due to variation in intensity of intermale sperm competition? Reprod Fertil Dev. 30:1434-1442.

Párraga M, del Mazo J. 2000. XYbp, a novel RING-finger protein, is a component of the $\mathrm{XY}$ body of spermatocytes and centrosomes. Mech Dev. 90:95-101.

Parsch J, Ellegren H. 2013. The evolutionary causes and consequences of sex-biased gene expression. Nat Rev Genet. 14:83-87.

Phifer-Rixey M, Nachman MW. 2015. Insights into mammalian biology from the wild house mouse Mus musculus. Elife 4.

Piasecka B, Lichocki P, Moretti S, Bergmann S, Robinson-Rechavi M. 2013. The Hourglass and the Early Conservation ModelsCo-Existing Patterns of Developmental Constraints in Vertebrates. PLoS Genet. 9:e1003476.

Pitnick S, Hosken DJ, Birkhead TR. 2009. Sperm morphological diversity. In: Birkhead TR, Hosken DJ, Pitnick S, editors. Sperm Biology. London: Academic Press. p. 69-149.

Ramm SA, Schärer L. 2014. The evolutionary ecology of testicular function: size isn't everything. Biol Rev. 89:874-888.

Robinson MD, McCarthy DJ, Smyth GK. 2010. edgeR: a Bioconductor package for differential expression analysis of digital gene expression data. Bioinformatics. 26

Rohlfs RV, Nielsen R. 2015. Phylogenetic ANOVA: The Expression Variance and Evolution Model for Quantitative Trait Evolution. Syst Biol. 64:695-708

Sabarís G, Laiker I, Preger-Ben Noon E, Frankel N. 2019. Actors with Multiple Roles: Pleiotropic Enhancers and the Paradigm of Enhancer Modularity. Trends Genet. 35:423-433.

Sánchez-Ramírez S, Weiss JG, Thomas CG, Cutter AD. 2021. Widespread misregulation of inter-species hybrid transcriptomes due to sex-specific and sex-chromosome regulatory evolution. PLoS Genet. 17:e1009409.

Sarver BAJ, Keeble S, Cosart T, Tucker PK, Dean MD, Good JM. 2017. Phylogenomic Insights into Mouse Evolution Using a Pseudoreference Approach. Genome Biol Evol. 9:726-739.

Schroeder CM, Valenzuela JR, Mejia Natividad I, Hocky GM, Malik HS. 2019. A Burst of Genetic Innovation in Drosophila Actin-Related Proteins for Testis-Specific Function. Mol Biol Evol. 37:757-772.

Schumacher J, Herlyn H. 2018. Correlates of evolutionary rates in the murine sperm proteome. BMC Evol Biol. 18:35.

Skinner BM, Johnson EEP, Bacon J, Affara NA, Rathje CC, Yousafzai G, Ellis PJI, Larson EL, Kopania EEK, Good JM. 2019. A high-throughput method for unbiased quantitation and categorisation of nuclear morphology.

Soumillon M, Necsulea A, Weier M, Brawand D, Zhang X, Gu H, Barthès P, Kokkinaki M, Nef S, Gnirke A, et al. 2013. Cellular Source and Mechanisms of High Transcriptome Complexity in the Mammalian Testis. Cell Reports. 3:2179-2190.

Streett DA, Petersen KR, Gerritsen AT, Hunter SS, Settles ML. 2015. expHTS: analysis of high throughput sequence data in an experimental framework. Proceedings of the 6th ACM Conference on Bioinformatics, Computational Biology and Health Informat- 
bioRxiv preprint doi: https://doi.org/10.1101/2021.08.04.455131; this version posted August 25, 2021. The copyright holder for this preprint (which was not certified by peer review) is the author/funder. All rights reserved. No reuse allowed without permission.

ics; Atlanta, Georgia: Association for Computing Machinery. p. 523-524.

Swanson WJ, Nielsen R, Yang Q. 2003. Pervasive Adaptive Evolution in Mammalian Fertilization Proteins. Mol Biol Evol. 20:18-20.

Swanson WJ, Vacquier VD. 2002. The rapid evolution of reproductive proteins. Nat Rev Genet. 3:137-144.

The UniProt Consortium. 2020. UniProt: the universal protein knowledgebase in 2021. Nucleic Acids Res. 49:D480-D489.

Thompson A, May MR, Moore BR, Kopp A. 2020. A hierarchical Bayesian mixture model for inferring the expression state of genes in transcriptomes. Proc Natl Acad Sci U S A. 117:19339-19346.

Thybert D, Roller M, Navarro FCP, Fiddes I, Streeter I, Feig C, Martin-Galvez D, Kolmogorov M, Janoušek V, Akanni W, et al. 2018. Repeat associated mechanisms of genome evolution and function revealed by the Mus caroli and Mus pahari genomes. Genome Res.

Turner LM, Chuong EB, Hoekstra HE. 2008. Comparative Analysis of Testis Protein Evolution in Rodents. Genetics. 179:2075-2089.

Vicens A, Borziak K, Karr TL, Roldan ERS, Dorus S. 2017. Comparative Sperm Proteomics in Mouse Species with Divergent Mating Systems. Mol Biol Evol. 34:1403-1416.

Vicoso B, Charlesworth B. 2009. Effective population size and the faster-x effect: An extended model. Evolution. 63:2413-2426.

Voolstra C, Tautz D, Farbrother P, Eichinger L, Harr B. 2007. Contrasting evolution of expression differences in the testis between species and subspecies of the house mouse. Genome Res. 17:42-49.

White-Cooper H, Doggett K, Ellis RE. 2009. The evolution of spermatogenesis. In: Birkhead TR, Hosken DJ, Pitnick S, editors. Sperm Biology. London: Academic Press. p. 151-183.

Whittle CA, Kulkarni A, Extavour CG. 2020. Absence of a Faster-X Effect in Beetles (Tribolium, Coleoptera). G3-Genes Genom Genet. 10:1125-1136.

Winter EE, Goodstadt L, Ponting CP. 2004. Elevated Rates of Protein Secretion, Evolution, and Disease Among Tissue-Specific Genes. Genome Res. 14:54-61.

Wray GA, Hahn MW, Abouheif E, Balhoff JP, Pizer M, Rockman MV, Romano LA. 2003. The evolution of transcriptional regulation in eukaryotes. Mol Biol Evol. 20:1377-1419.

Yang Z. 2007. PAML 4: Phylogenetic Analysis by Maximum Likelihood. Mol Biol Evol. 24:1586-1591.

Yapar E, Saglican E, Dönertaş HM, Özkurt E, Yan Z, Hu H, Guo S, Erdem B, Rohlfs RV, Khaitovich P, et al. 2021. unpublished data. https:// wwwbiorxivorg/content/101101/010553v2. last accessed July 12, 2021.

Zhao L, Saelao P, Jones CD, Begun DJ. 2014. Origin and Spread of de Novo Genes in Drosophila melanogaster Populations. Science. 343:769-772. 